\title{
Nonfrontal Expression Recognition in the Wild Based on PRNet Frontalization and Muscle Feature Strengthening
}

\author{
Tianyang Cao $\mathbb{D}^{1,2}$ Chang Liu $\left.\mathbb{D}\right)^{1,2}$ and Jiamin Chen $\mathbb{D}^{1,2}$ \\ ${ }^{1}$ Aerospace Information Research Institute, Chinese Academy of Sciences, Beijing 100190, China \\ ${ }^{2}$ School of Electronic, Electrical and Communication Engineering, University of Chinese Academy of Sciences, \\ Beijing 100049, China \\ Correspondence should be addressed to Tianyang Cao; cao_tian_yang@sina.cn, Chang Liu; tuengineer@qq.com, and \\ Jiamin Chen; jmchen@mail.ie.ac.cn
}

Received 3 November 2020; Revised 20 March 2021; Accepted 30 May 2021; Published 12 July 2021

Academic Editor: Georgios I. Giannopoulos

Copyright (c) 2021 Tianyang Cao et al. This is an open access article distributed under the Creative Commons Attribution License, which permits unrestricted use, distribution, and reproduction in any medium, provided the original work is properly cited.

\begin{abstract}
Nonfrontal facial expression recognition in the wild is the key for artificial intelligence and human-computer interaction. However, it is easy to be disturbed when changing head pose. Therefore, this paper presents a face rebuilding method to solve this problem based on PRNet, which can build 3D frontal face for 2D head photo with any pose. However, expression is still difficult to be recognized, because facial features weakened after frontalization, which had been widely reported by previous studies. It can be proved that all muscle parameters in frontalization face are more weakened than those of real face, except muscle moving direction on each small area. Thus, this paper also designed muscle movement rebuilding and intensifying method, and through 3D face contours and Fréchet distance, muscular moving directions on each muscle area are extracted and muscle movement is strengthened following these moving directions to intensify the whole face expression. Through this way, nonfrontal facial expression can be recognized effectively.
\end{abstract}

\section{Introduction}

Nonfrontal facial expression recognition (FER) in the wild is very important for artificial intelligence (AI), and it is the key for human-computer interaction (HCI) [1]. If HCI system wants to act as a real human to communicate with its human clients, the computer needs to recognize the clients' facial expression and detect their emotion effectively [2]. However, at present, FER methods have not yet reached the level of practice, and most of them could only recognize expression on front face; the FER methods for nonfrontal faces are still under research [3].

However, in the wild, FER is challenged by nonfrontal faces, illumination variations, and registration errors [4]. The nonfrontal faces are caused by head turning and pitching and camera viewpoints changing, and these would be easy to deform face shape significantly and cause FER errors [5]. In deformed facial images, the important features for expression recognition, including the appearance and position of brows, eyes, cheeks, and mouth, are much different from those of front face and cause FER error seriously. The other challenges for FER are also related to these interferences in nonfrontal faces [6]. If illumination is poor, especially when head is turning, some face parts cannot be illuminated enough and might be dark and blurry in the photos; FER error may be caused [7]. For the deformed faces, the matching with standard front face is also impossible. Because geometrical relationship among these key points on deformed face image might be deformed and might be much different from that of front face, the traditional FER methods would be disturbed seriously [8].

In order to solve these problems of FER in the wild, there are usually three ways: extracting facial key points as expression features, extracting the expression features on the whole or local face, and establishing the relationship of different poses and expression.

The facial key points, such as the points on the brows, eyes, and mouth, are very useful for FER, because they are very sensitive to facial muscle movement [9]. The newest studies for this issue concentrate on introducing self-study 
method to build model for key points localization; for example, the study in [10] established hierarchical probabilistic model to extract key points on the deformed face. As in-thewild is very important for computer vision, traditional methods are also improved for extracting facial key points in nonfrontal faces, for example, training Active Shape Model (AAM) in-the-wild to eliminate the disturbance caused by head pose changing in [11]. While facial key points can be localized exactly, the expression recognition can still be disturbed, because FER models through key points mainly rely on the geometrical relationship among these points. While the relationship among 3D key points geometrical projection on the $2 \mathrm{D}$ image would lose a lot of information, the geometrical projection of the same expression on every facial photo is much different when head poses are different. This problem might cause FER error, especially when head pose changes significantly.

For the expression features on the whole or local face, they are more adaptable for FER in the wild. Comparing key points, much more expression information can be gotten from face areas, for example, eyes, brows, cheeks, and mouth. Its core is to extract the local or whole facial features of different poses. The newest deep learning methods are very suitable for this issue [12]. The study in [13] proposed weighted mixture deep networks fusing facial grayscale images features and their corresponding LBP images for FER. The facial expression features are extracted through a pretrained model (VGG16 model on ImageNet). The study in [14] proposed Weighted Center Regression Adaptive Feature Mapping (W-CR-AFM); this network could finetune parameters to gain better recognition accuracy in the specific applications, and these misclassified samples and new samples can be corrected or reformulated. The authors also proposed preprocessing method to assist the AFM by extracting the precise face images based on Neighbor-Center Difference Image (NCDI) method. The study in [15] proposed Multichannel Pose-aware Convolution Neural Networks (MPCNN) for nonfrontal faces expression recognition; this network is combined by three parts: multichannel feature extraction, jointly multiscale feature fusion, and pose-aware recognition. However, there are still some problems. As the result of $3 \mathrm{D}$ face solid projection, the spatial information loss in 2D facial photos is still serious with different head poses. The expression features deformation and displacement are different in every nonfrontal face image and may cause FGR error.

Compared the facial feature points and regional expression features, establishing the relationship of different poses and expression may be the most effective way for nonfrontal faces expression recognition. For example, one common way is to establish the transformation model between front face and nonfrontal faces to frontalize nonfrontal faces. FER in the wild would be much convenient according to these frontalization faces. Although the lack of face deep information in the $2 \mathrm{D}$ photo would cause some difficulty for face frontalizing, the deep learning method, especially these deconvolution networks, can build effective frontalization model for nonfrontal faces. The typical methods include Generative Adversarial Nets (GAN), 3D morphable model (3DMM), 3D Dense Face Alignment (3DDFA), and Position Map Regression Network (PRNet). GAN could transform nonfrontal 2D face image into $2 \mathrm{D}$ front face [16]. The study in [17] introduced GAN to gain the front faces of nonfrontal faces and using these frontalization faces for expression recognition. 3DMM [18], 3DDFA [19], and PRNet [20] introduce another way for face frontalization; they could directly build the 3D faces for nonfrontal faces, and the front face can be gotten through the projection of $3 \mathrm{D}$ face. The $3 \mathrm{D}$ face could contain much more information and improve FER accurate.

Therefore, in recent years, the deep learning, especially $\mathrm{CNN}$, has been extensively applied in expression recognition. These networks can be designed to recognize expression with any pose. The study in [14] presented Multichannel Pose-aware Convolution Neural Networks (MPCNN) to extract facial features; they consisted of multichannel feature extraction, joint multiscale feature fusion, and pose-aware recognition. The study in [21] proposed Region Attention Network (RAN), which is a region-based deep attention architecture. This network can integrate visual clues from regions and whole faces to capture the important facial regions, and the weights of this network can be refined adaptively. The study in [12] used weighted mixture deep networks (VGG16 model) to improve traditional local binary pattern for expression. The study in [13] proposed Weighted Center Regression Adaptive Feature Mapping (W-CR-AFM) to eliminate misclassified samples or add new samples to improve model accurate. The study in [22] improved convolution neutral network with attention mechanism, and two networks were proposed: patch-based ACNN (pACNN) and global-localbased ACNN (gACNN). These networks can focus on the most discriminative unoccluded face regions and design gate unit that could weigh each region adaptively. The study in [23] proposed parallel feature extraction-based lightweight CNN, named as eXnet (Expression Net), which can combine accuracy and a smaller number of parameters. The study in [4] proposed and combined three different novel CNN models together. The first one is consisting of six depth-wise separable residual convolution modules and can solve the problem of complex topology and overfitting. The second one has dual-branch and could perform extraction. Traditional LBP features and deep learning features are parallel. The third one could overcome the shortage of training samples based on transfer learning technique. The study in [24] proposed island loss (IL-CNN) to improve $\mathrm{CNN}$, which can enlarge interclass differences and reduce intraclass variations simultaneously. The study in [25] proposed identity-aware convolutional neural network (IACNN). The authors designed identity-sensitive contrastive loss to recognize identity-invariant expression for particular people.

However, all the above methods cannot accomplish FER in the wild effectively. For the facial muscle movement, which can show the direct expression directly, it might be weakened on the nonfrontal faces or its frontalization and cause FER error. Some important parameters of muscle movement, such as the moving zone and the extent of 
contraction or relaxation, cannot be calculated exactly. This is a serious problem for frontalization face and had been widely reported in the previous studies. In this paper, based on the theory of " $N: q$ rule" of parameter estimates $[26,27]$ and face finite element analysis (FEM) [28-30] and muscle structure anatomy [31,32], it would be proved that all muscle parameters in frontalization face are more weakened than those of real face, except muscle moving direction on each small area.

In order to solve these problems, this paper presents a more useful method for accomplishing FER in the wild. It could rebuild face for frontalizing and intensify muscle movement to strengthen expression features on frontalization face and improve expression accuracy more effectively.

Firstly, deep learning method PRNet is introduced. This network could extract nonfrontal faces in the photos and build their $3 \mathrm{D}$ face for frontalizing. In consideration of human face which is symmetrical, the front face can be established through the half face toward camera and its mirror in the 3D face. In order to analyze model accuracy for the face muscle, 3D face is divided as skull model and muscular layer. According to the theory of " $N: q$ rule" of parameter estimates [26, 27], it can be proved that skull model can be rebuilt exactly. Meanwhile, for the muscle model, according to the theory of muscular structure and cell shape, 3D face can only estimate and show muscular moving direction on each small area in the face, and the other parameters of muscle movement cannot be estimated effectively, and facial expression features on 3D frontalization face are weakened significantly. Therefore, face contour model and muscle movement strengthening model are proposed for intensifying expression features. For face contour model, face contour and Fréchet distance are proposed in this paper for dividing face into many small areas and extracting muscular moving direction in each area. Through muscle movement strengthening model, each area is given a 3D Gauss model that can follow the muscle moving direction to simulate and strengthen muscle movement. These Gauss strengthening models in different face areas could partially overlap each other to strengthen muscle contraction or relaxation in the whole frontalization face image for intense facial features.

In the test, the famous MEGVII Face++ facial expression recognition platform, which is also able to recognize expression in the wild, is used for comparison. Face++ recognizes the expression in the original nonfrontal faces and frontalization faces and expression strengthened frontalization faces, respectively. Test result shows that the method proposed in this paper could enlarge the facial feature effectively and recognition accuracy in the strengthened frontalization face is much higher than that of original face.

\section{Method Design}

The structure of nonfrontal facial expression recognition method is shown in Figure 1. It is composed of two parts: face frontalization through PRNet 3D model and muscle movement strengthening and expression strengthening model.

For every face in the 2D photo, PRNet could build special 3D face for each of them. The frontalization faces can be gotten from these $3 \mathrm{D}$ faces, and muscle movement can also be extracted from these $3 \mathrm{D}$ faces.

For the nonfrontal face, it is inevitable that facial feature in frontalization face is more weakened than that of real front face. So, this paper presents a model to strengthen muscle movement and facial expression features and improve FER accuracy.

\subsection{Face Frontalization and Facial Muscle Movement Extraction}

2.1.1. Face Frontalization. PRNet could output the 3D face just through its $2 \mathrm{D}$ head photo. The core of PRNet is ten residual blocks, and their self-study and modeling ability is much stronger than those of ordinary convolution layers. These blocks could extract $2 \mathrm{D}$ face image features and transfer these features into $8 \times 8 \times 512$ feature maps. 17 transposed deconvolution layers use these features to decode and generate the $256 \times 256 \times 3$ position map, named as UV position map by the PRNet authors [20]. This UV position map is the $3 \mathrm{D}$ face. After projecting and frontalizing this $3 \mathrm{D}$ face, the front face can be gotten.

In order to recognize facial expression effectively, the face symmetry is taken advantage of to build front face. As the left and right faces are symmetric, the muscle movement and expression on these two sides are also the same. So, the face frontalization is just using the half 3D face, which is toward the camera and is less deformed and much clearer on the photo, to build front face. Its mirror can substitute the other half face that is more seriously deformed and compressed in the original 2D photo. After 3D face rotation transform and projection, the frontalization face can be gotten.

Figure 2 shows the image processing of a side face frontalization through its left $3 \mathrm{D}$ face and its mirror. The half face toward camera can be extracted according to the head pose resolved by the PRNet.

Through this method, the expression of nonfrontal faces can be recognized, for example, in Figures 3-5. Figure 3 shows the original sad face and its frontalization.

In the experiment, the famous Face++ recognition platform is taken advantage of to test our frontalization method. As shown in Figure 4, there are 7 horizontal bars on the right of Face++ platform. They show the prediction confidence of seven expressions. From top to bottom, these are "happy," "neutral," "surprise," "sad," "disgust," "angry," and "fear" and are noted in English. The most probable expression recognition result is orange-colored.

The original face of turning and bowing head is error recognized as neutral, as shown in Figure 4. Meanwhile, after face frontalization by our method, "sad|" can be recognized effectively, as shown in Figure 5.

While there is still a problem for face frontalization, as the muscle movement for expression in frontalization face is 


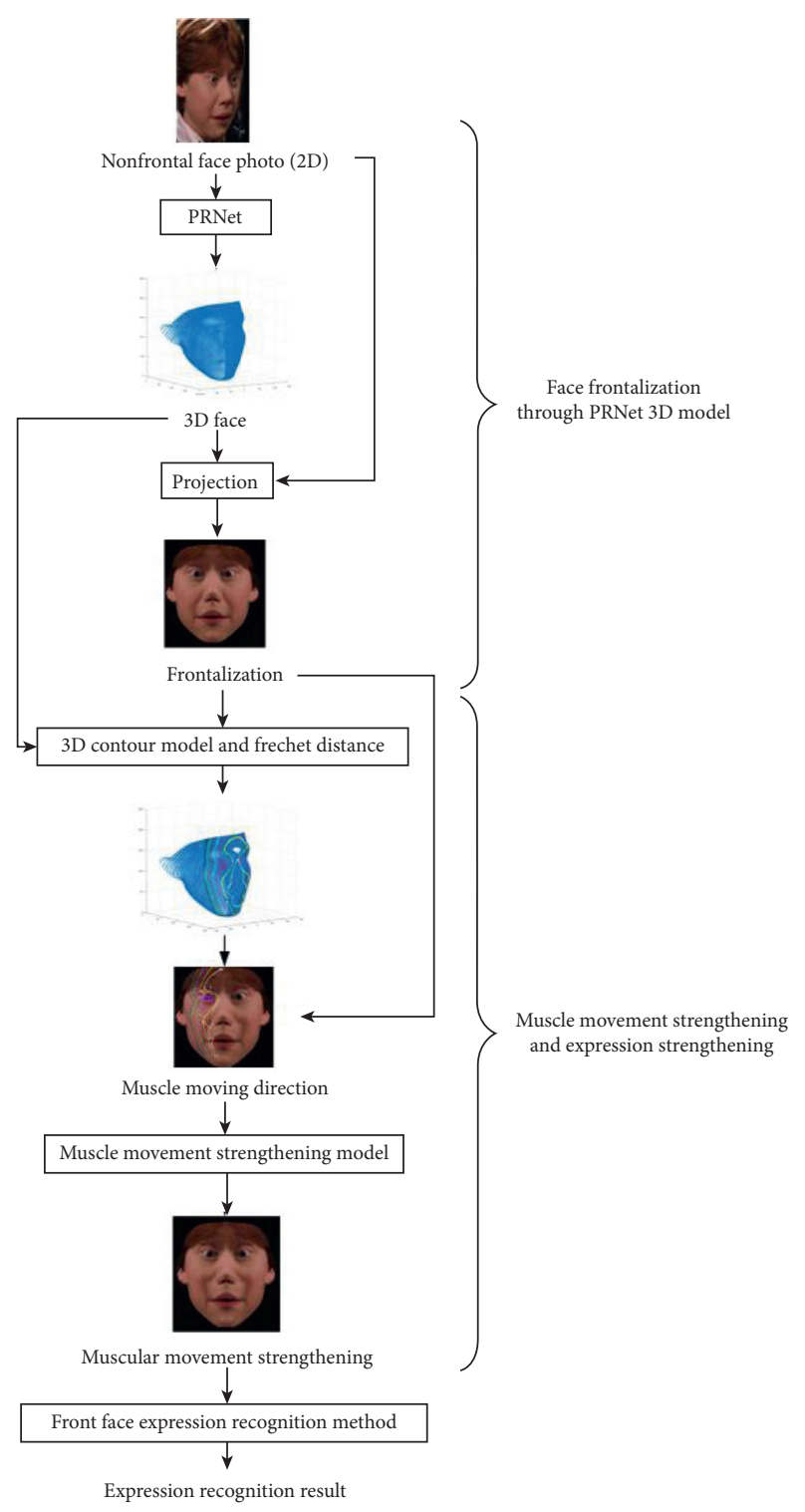

FIgURE 1: The image processing of this method.

more weakened than that of real front face, almost all of the frontalization researches are bothered by this problem. As shown in Figure 6, Figures 6(a) and 6(b) are the front and nonfrontal face images of the former US president Richard M. Nixon, and Figure 6(c) is the frontalization of Figure 6(b). It can be shown that the facial features and muscle movement on frontalization face are more weakened than original face significantly.

There are two reasons for this problem: One is that because some facial organ areas are compressed in the nonfrontal-view face; their resolution is very low in the nonfrontalization face image and still blurry in the frontalization image, for example, the brow, eye, nose, and mouth in the side face which is not toward the camera. The other is that muscle movement cannot be rebuilt effectively in the frontalization model, and this is the main interference for expression recognition.

Therefore, we would solve these problems by strengthening facial muscular movement on the $3 \mathrm{D}$ face to intensify facial expression feature.

2.1.2. Accuracy Analysis for Facial Muscle Movement in 3D Face. In order to analyze the accuracy of muscle movement on $3 \mathrm{D}$ face, the real human head is decomposed into two parts: skull model (blue area) and muscular layer model (red area), as shown in Figure 7. The characterization of expression on the skin is produced by the muscular layer. So the 3D face building through PRNet can also be equivalent to build these two models through PRNet.

The fitting accuracy of PRNet for 3D skull and 3D muscle can be analyzed from two aspects: PRNet fitting ability distribution for them and the number of their parameters that need to be estimated. For example, if fitting ability for the facial muscle is weak and muscular parameters are numerous, it is impossible to build precise muscular movement in the $3 \mathrm{D}$ face.

The distribution of PRNet fitting ability for skull and muscular movement can be discussed through the loss function, which is the core of PRNet training to build 3D face; and, according to the theory of Finite Element Method (FEM), face can be divided into many small areas for analysis. For each small area $i, P(x, y)=P_{H}(x, y)+$ $P_{E, i}(x, y)$ and $P_{H}(x, y)$ and $P_{E, i}(x, y)$ is the real value of skull and muscular UV maps output by PRNet. Therefore, for each self-study epoch, the loss function for driving PRNet training is

$$
\begin{aligned}
& \text { loss } \\
& =\sum\|P(x, y)-\widetilde{P}(x, y)\| \cdot W(x, y) \\
& =\sum\left\|P_{H}(x, y)-\left(1-r_{i}\right) \widetilde{P}(x, y)+P_{E, i}(x, y)-r_{i} \widetilde{P}(x, y)\right\| \cdot W_{i}(x, y),
\end{aligned}
$$

where $\widetilde{P}(x, y)$ is PRNet output and it is named as UV maps transformed from $3 \mathrm{D}$ face coordinates, $P(x, y)$ represents the UV maps of real $3 \mathrm{D}$ face, $W_{i}(x, y)$ is the weight of this area in loss function, and $r_{i}$ is the ratio between muscle and skull: $r_{i}=\left(\left(P_{E, i}(x, y)\right) /\left(P_{H}\right.\right.$ $\left.\left.(x, y)+P_{E, i}(x, y)\right)\right)$.
Because the size and volume of skull are much larger than those of muscle in the skin, it can be deduced that $P_{H}(x, y) \gg P_{E, i}(x, y)$. So the ratio of output for skull $\left(1-r_{i}\right)$ is much larger than that of face muscular $r_{i}$, $\widetilde{P}(x, y)\left(1-r_{i}\right) \gg \widetilde{P}(x, y) r_{i}$. This means that PRNet would distribute most of its ability to fit skull and revise skull model 


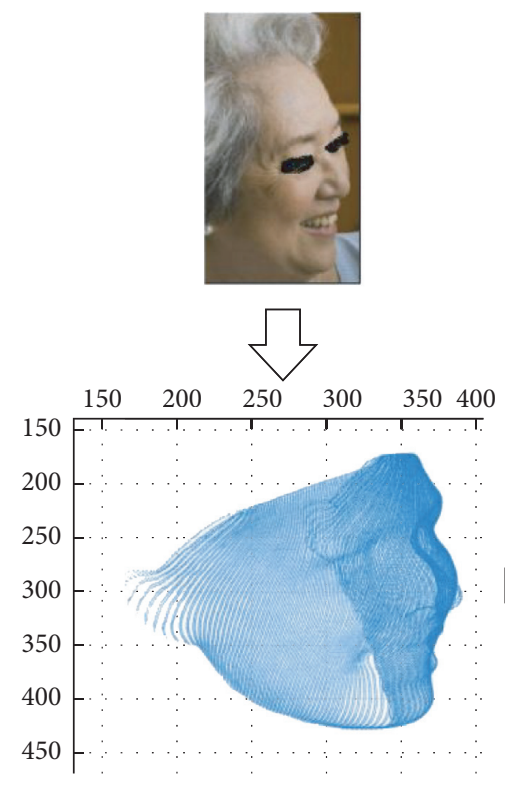

3D face model

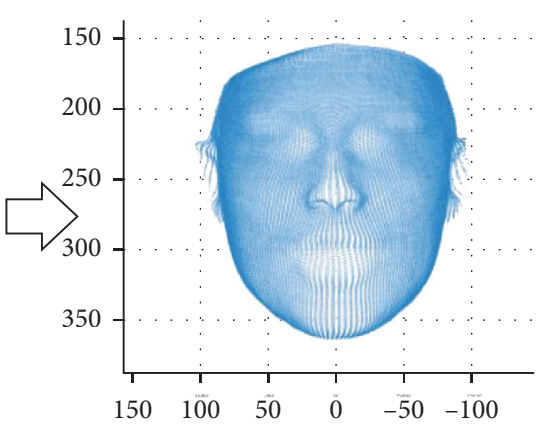

Projecting and frontalizing
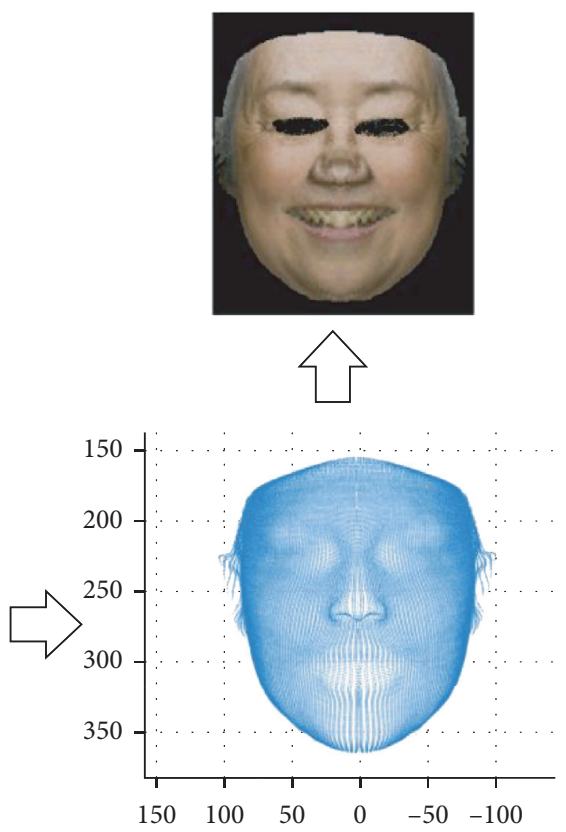

Left half face and its mirror

FIGURE 2: Face frontalization through PRNet and half face.

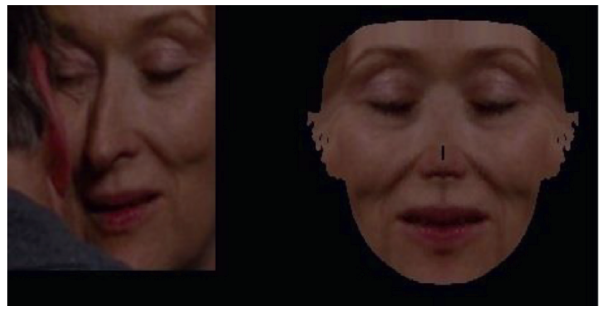

(a)

(b)

Figure 3: Original sad face (a) and its frontalization (b).
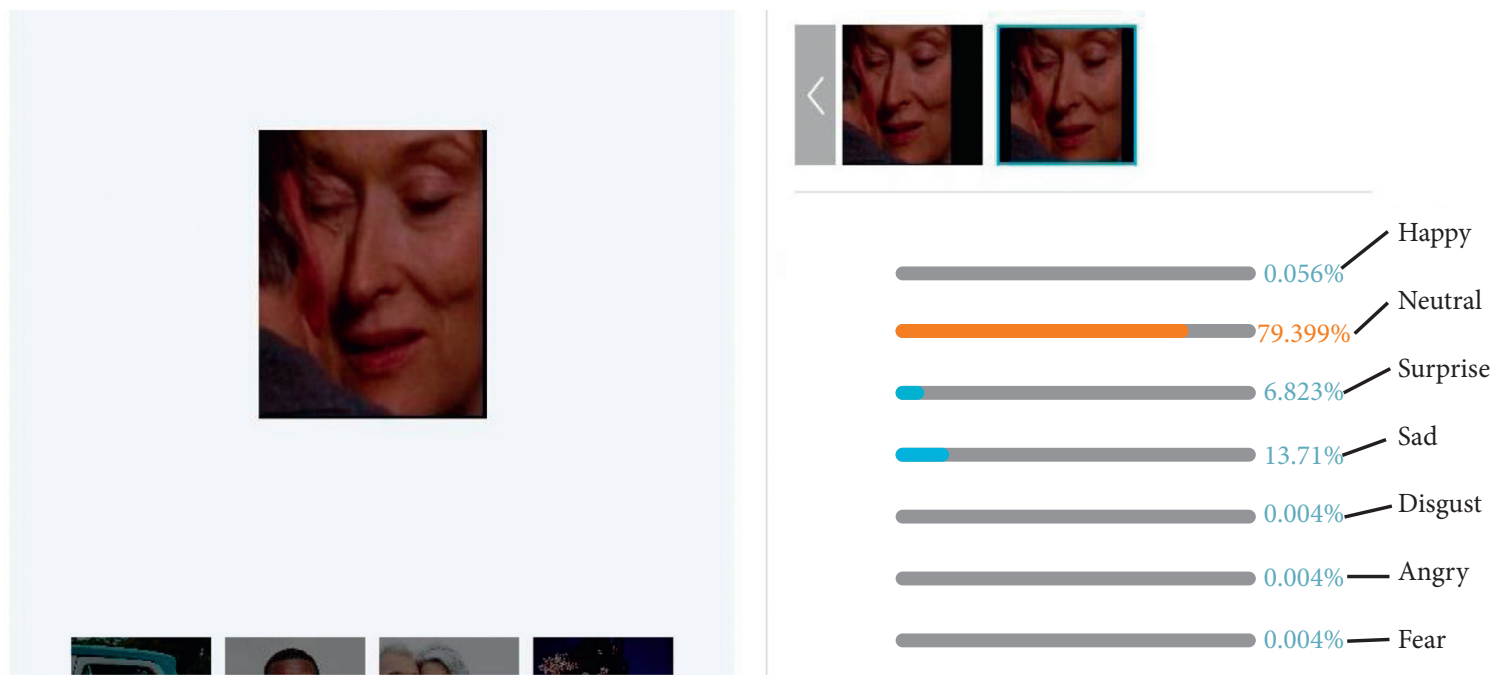

FIGURE 4: Original sad face is mistakenly recognized as "neutral." 


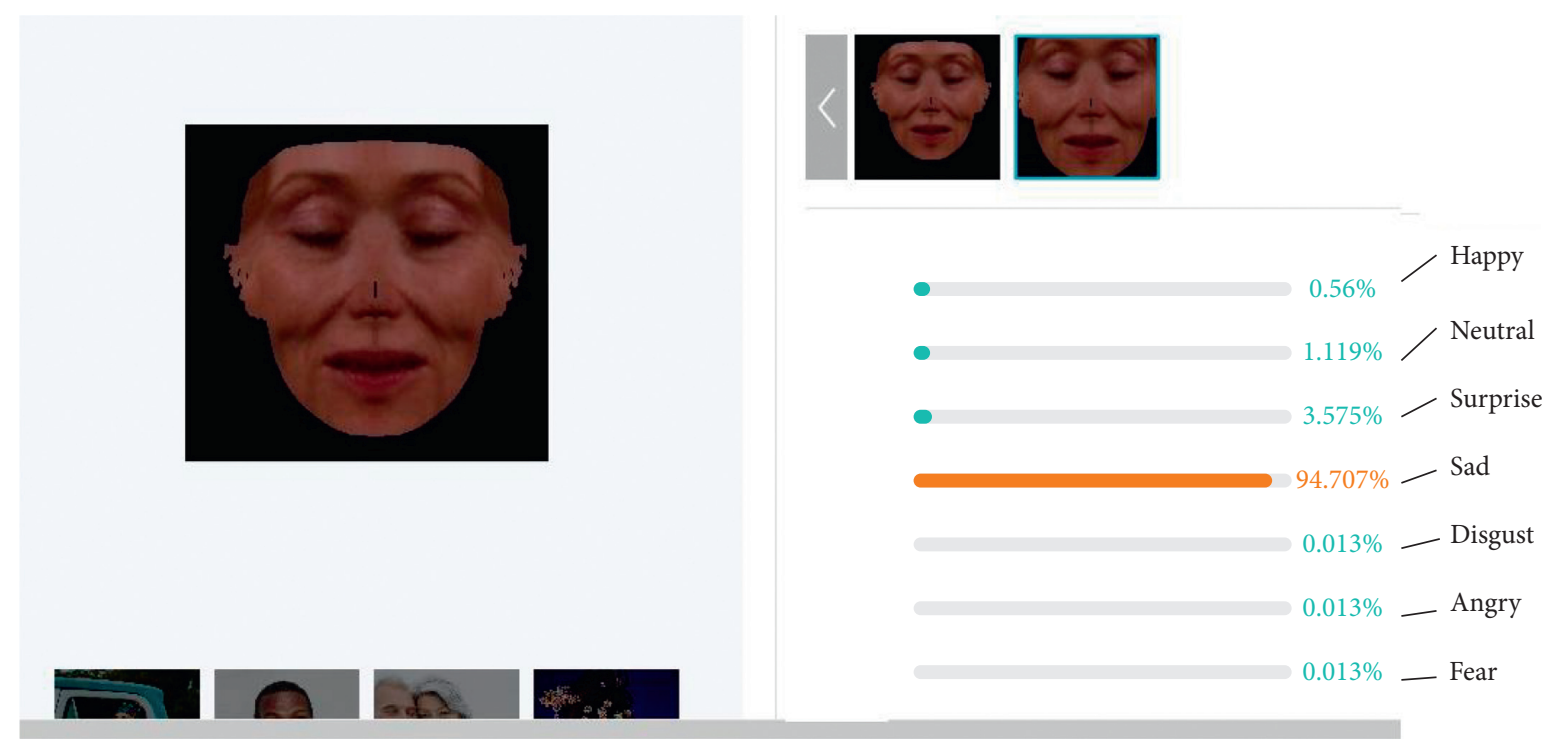

FIgURE 5: The frontalization face is correctly recognized as "sad."

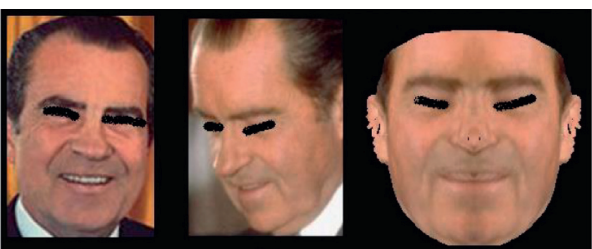

(a)

(b)

(c)

FIGURE 6: The difference between real front face and frontalization face. (a) Real front face, (b) side face, and (c) its frontalization face.

error. So, for the facial muscle, the remaining PRNet fitting ability is very less and fitting error may be large. This is a disadvantage for fitting muscle movement and weakens the expression feature on the 3D face.

What was worse, for the $3 \mathrm{D}$ face parameter estimation, some important parameters in muscle movement model cannot be estimated effectively as that of skull model. This can be proved through the theory of Finite Element Method (FEM) [28]:

(1) The Parameters Fitting Error for Skull Model. For the skull model, it can be descripted as follows:

$$
\left(X_{H}, Y_{H}, Z_{H}\right)=F_{\text {skull }}\left(I_{x}, I_{y}\right) \text {. }
$$

The relationship among these $3 \mathrm{D}$ points $\left(X_{H}, Y_{H}, Z_{H}\right)$ follows the structure of skull geometric. According to the FEM theory, the skull is composed of a lot of finite elements, and the number is $n_{0}$. For each element, its model contains $n_{1}$ parameters $p_{1, i}, p_{2, i}, \ldots, \mathrm{p}_{n 1, i}$ and can be shown as follows:

$$
\left(X_{H}, Y_{H}, Z_{H}\right)=F_{\text {skull, } i}\left(I_{x}, I_{y}, p_{1, i}, p_{2, i}, \ldots, p_{n 1, i}\right) .
$$

Function (3) shows the model of the No. $i$ element. If the PRNet could estimate the parameters of each skull element exactly, it could build skull model exactly.
According to the theory of " $N: q$ rule" of parameter estimation $[26,27]$, sample number needs to be much larger than parameter number. The study in [26] suggested that the ratio should be at least larger than $5: 1$, and the study in [27] suggested that the ratio should be larger than $10: 1$ or $20: 1$. So, for the $3 \mathrm{D}$ face built by PRNet, because it contains $256 \times$ 256 fix points on the head, it can be seen as using $n_{0}=$ $256 \times 256$ elements to establish skull model, and total parameter number is $n_{0} \times n_{1}=256 \times 256 \times n_{1}$. For deep learning network training by massive faces database, input sample number for training PRNet must be much larger than $20 \times 256 \times 256 \times n_{1}$, and all the facial element parameters can be resolved. So, for the "neutral" expression, as muscle movement is very less, its $3 \mathrm{D}$ face can be built exactly, as shown in Figure 8.

The front face for each nonfrontal face image is the projection of front 3D face, which can be gotten by rotating 3D nonfrontal face through Euler matrix:

$$
\left(\begin{array}{c}
X_{H, i} \\
Y_{H, i} \\
Z_{H, i}
\end{array}\right)=A_{k, i}(\text { heading, pitch, roll })\left(\begin{array}{c}
X_{H, k} \\
Y_{H, k} \\
Z_{H, k}
\end{array}\right) \text {. }
$$

The Euler matrix $A$ (heading, pitch, roll), composed by heading and pitch and roll angles, would be gotten by comparing the arrays of standard front 3D face points and 3D face points in the wild by the Least-Square Method. PRNet provides a special function "frontalize ( )" for projecting and getting front face. So, as long as the number of input face samples is much larger than the number of skull parameter, the skull model can be fitted exactly by PRNet.

(2) The Parameter Fitting Error for Muscular Model. While, for the model of muscular layer, there may be some fitting error, muscular model is more complicated than skull model, and not all parameters of muscle movement can be resolved through PRNet. 


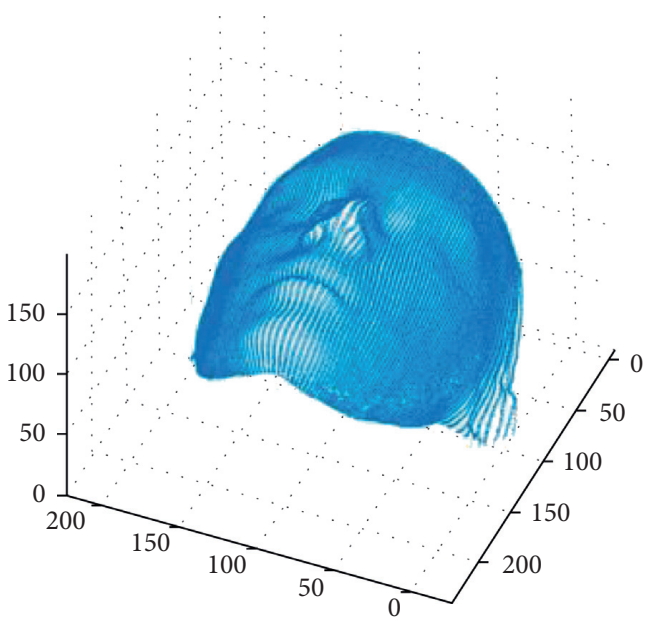

(a)

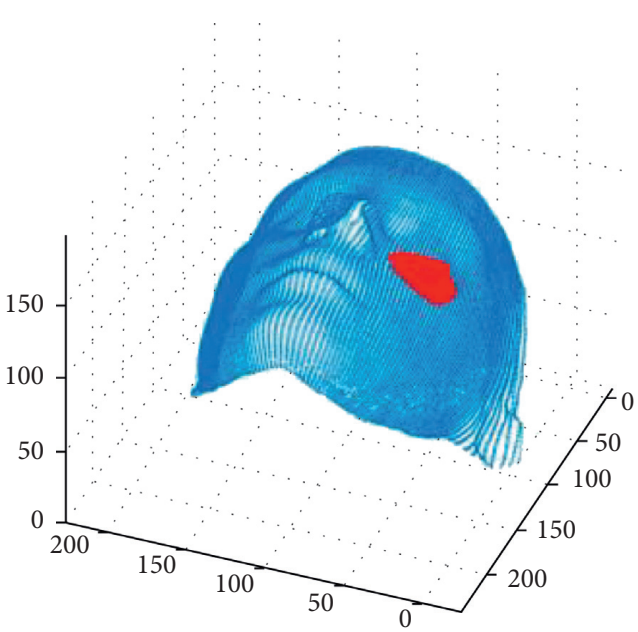

(b)

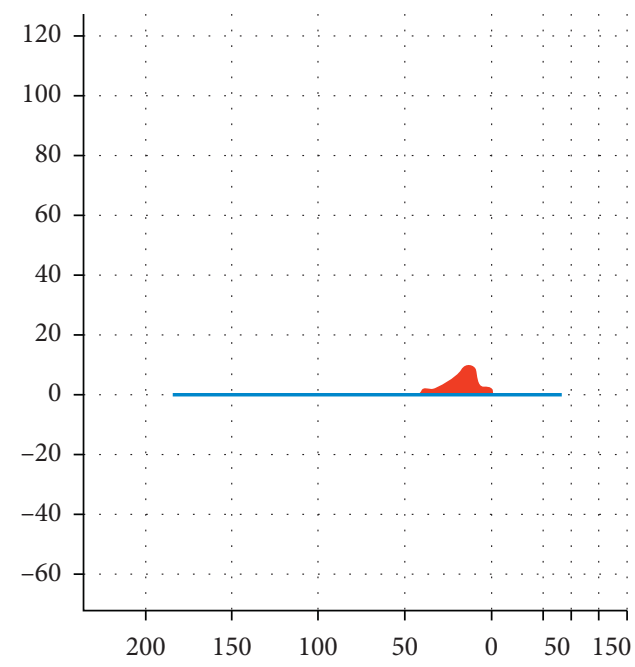

(c)

Figure 7: The separation of head model. (a) Skull model. (b) Muscular layer for expression (red). (c) Muscular layer profile (red).

According to the FEM theory [28] and facial action coding theory [29], facial muscular expression can also be divided into many small expression areas, given that total small area number is $n_{m}$. When receiving emotion signal from brain, the muscle in some areas would contract or relax and control the face skin to make expression (as the muscular layer profile shown in Figure 2(c)). The key for descripting muscle movement is that building model to give the different contraction and relaxation value to different skin points, as shown in Figure 9.

The real facial muscle movement is very complicated. Reference [30] presented the important muscular moving parameters, for example, zone of influence, moving direction and displacement of each point, and the different fitting model for the muscles of eye, mouth, cheek, and so forth.
Reference [31] proposed an image deformation method based on moving least squares for whole face deform, using a group of linear functions including affine, similarity, and rigid transformations.

For simplifying the model, in this paper, the face is divided into may small areas to decompose the whole face muscle movement. In each small area, the muscular movement is simplified as 3D Gauss model. As shown in Figure 10, this model includes three main parameters to describe muscle movement: muscle moving direction $\theta_{m}$, muscle area extent $d_{m}$ (diameter), and the inclination angle $\alpha_{m}$ of 3D face. $d_{m}$ and $\alpha_{m}$ could describe the extent of muscle contraction and relaxation. The muscle movement can be strengthened by enlarging area $d_{m}$ or decreasing 


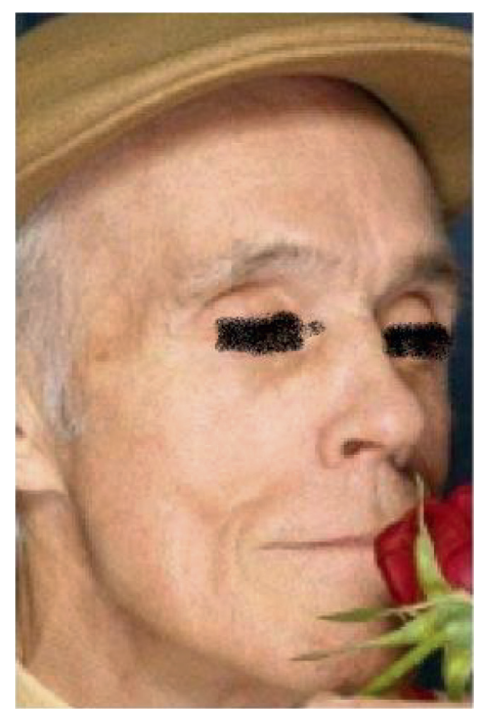

(a)

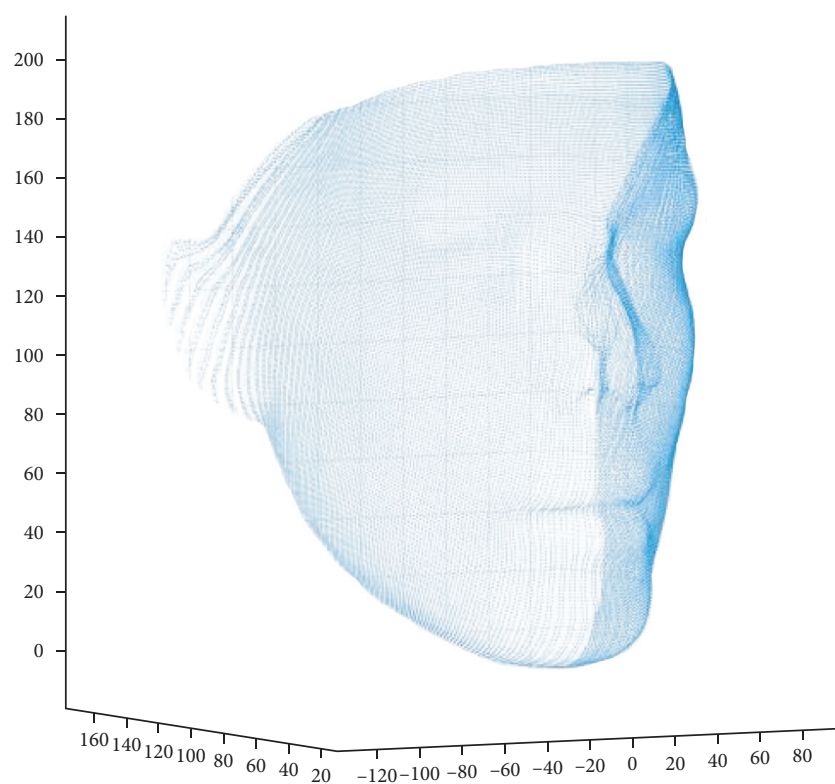

(b)

Figure 8: Original "neutral" face (a) and its 3D face with $256 \times 256$ elements (b).

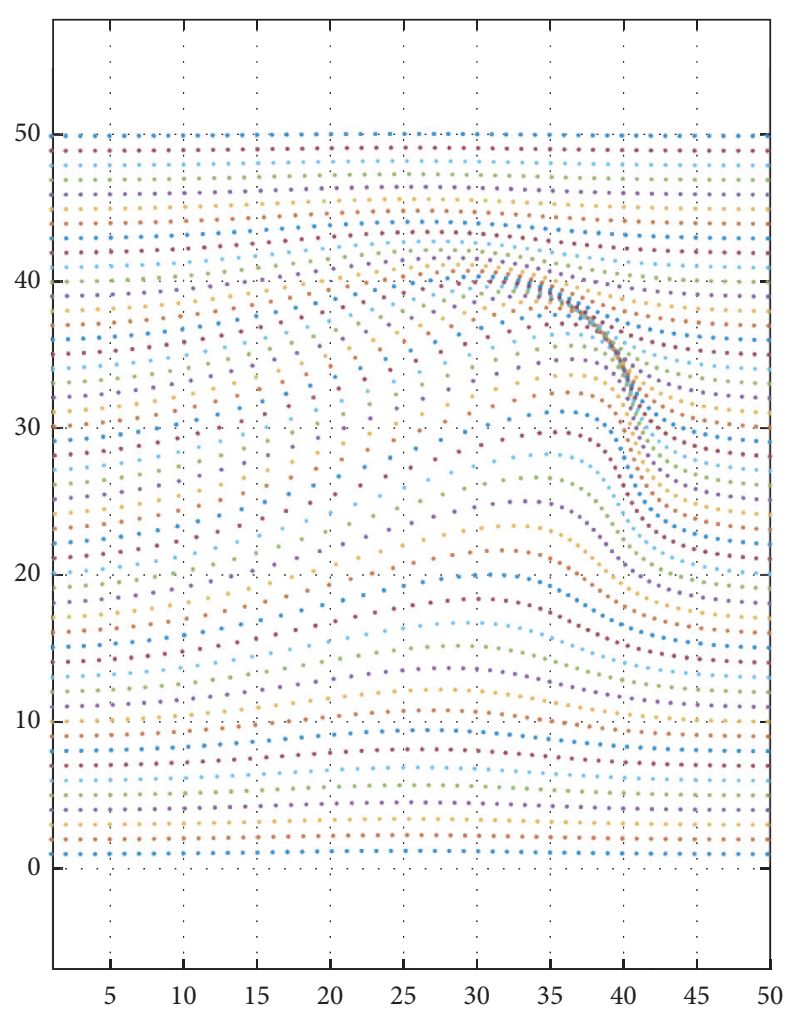

FIGURE 9: Muscle movement simulated by computer. 


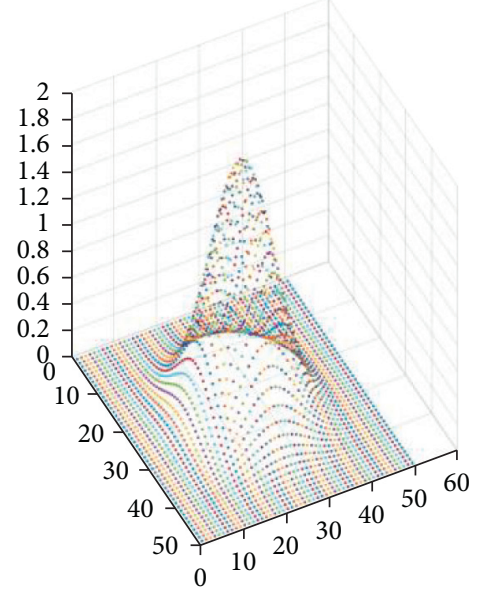

(a)

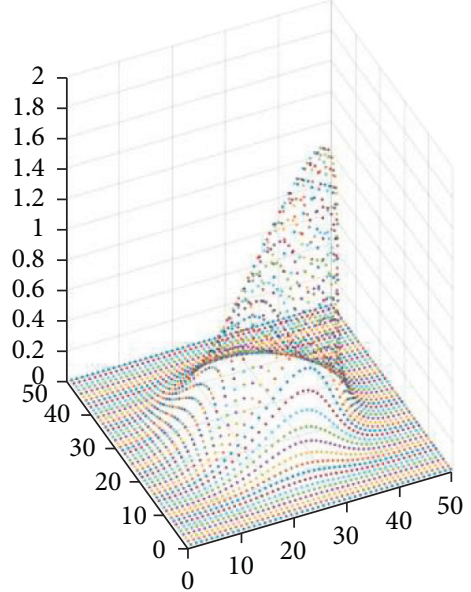

(b)

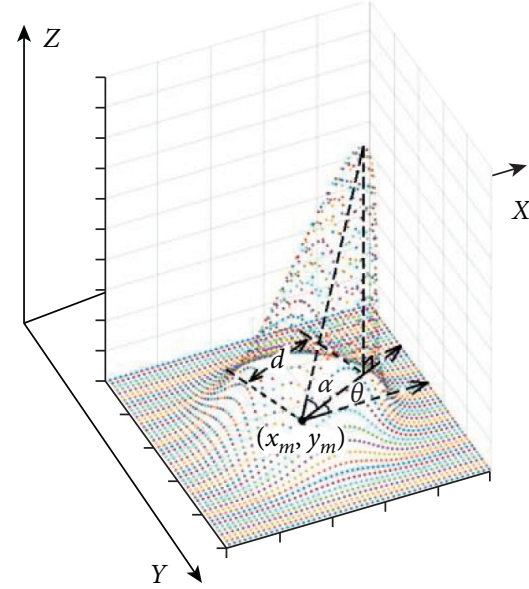

(c)

Figure 10: 3D Gauss muscular model. (a) Gauss model. (b) Inclining Gauss model to show muscular moving. (c) The main moving parameters.

inclination angle $\alpha_{m}$ (when $\alpha_{m} \approx 0$, the inclination is maximum and muscle movement is maximum, as shown in Figure 10(c)).
For the No. $m$ muscular small area, whose center is $\left(x_{m}, y_{m}\right)$, its Gauss model can be described as follows:

$$
\left(X_{m, i}, Y_{m, i}, Z_{m, i}\right)=F_{m u s, m}\left(x_{m}, y_{m}, \theta_{m}, d_{m}, \alpha_{m}, X_{m, 0}, Y_{m, 0}, Z_{m, 0}\right),
$$

where $\left(X_{m, 0}, Y_{m, 0}, Z_{m, 0}\right)$ is face point position of "neutral" and $\left(X_{m, i}, Y_{m, i}, Z_{m, i}\right)$ is face point new position in facial expression.

If parameters $\theta_{m}, d_{m}, \alpha_{m}$ for each area can be resolved by deep learning network PRNet exactly, the muscular model can be gotten. However, not all of them can be resolved by parameter estimating through sample images.

As the muscle structure in different areas is different, parameters $\theta_{m}, d_{m}, \alpha_{m}$ are the functions of muscle area center $\left(x_{m}, y_{m}\right)$, and they are changed with the displacement of $\left(x_{m}, y_{m}\right)$; the functions are as follows:

$$
\left\{\begin{array}{l}
\theta_{m}=F_{m u s, \theta, m}\left(I_{x}, I_{y}, p_{m, \theta, 1}\left(x_{m}, y_{m}\right), p_{m, \theta, 2}\left(x_{m}, y_{m}\right), \ldots, p_{m, \theta, n \theta}\left(x_{m}, y_{m}\right)\right), \\
d_{m}=F_{m u s, d, m}\left(I_{x}, I_{y}, p_{m, d, 1}\left(x_{m}, y_{m}\right), p_{m, d, 2}\left(x_{m}, y_{m}\right), \ldots, p_{m, d, n d}\left(x_{m}, y_{m}\right)\right), \\
\alpha_{m}=F_{m u s, \alpha, m}\left(I_{x}, I_{y}, p_{m, \alpha, 1}\left(x_{m}, y_{m}\right), p_{m, \alpha, 2}\left(x_{m}, y_{m}\right), \ldots, p_{m, \alpha, n \alpha}\left(x_{m}, y_{m}\right)\right),
\end{array}\right.
$$

where $p_{m, \theta, 1}\left(x_{m}, y_{m}\right), p_{m, \theta, 2}\left(x_{m}, y_{m}\right), \ldots, p_{m, \theta, n \theta}\left(x_{m}, y_{m}\right)$, $p_{m, d, 1}\left(x_{m}, y_{m}\right), p_{m, d, 2}\left(x_{m}, y_{m}\right), \ldots, p_{m, d, n d}\left(x_{m}, y_{m}\right), \quad$ and $p_{m, \alpha, 1}\left(x_{m}, y_{m}\right), p_{m, \alpha, 2}\left(x_{m}, y_{m}\right), \ldots, p_{m, \alpha, n \alpha}\left(x_{m}, y_{m}\right)$ are the subparameters of the models of parameters $\theta_{m}, d_{m}, \alpha_{m}$; and their numbers are $n_{\theta}, n_{d}, n_{\alpha}$, given that the total number of small muscle areas in the face is $n_{M}$.

The estimation of parameters $d_{m}$ and $\alpha_{m}$, which are used to show the extent of muscle movement, may be difficult. Their subparameters are relative to area center $\left(x_{m}, y_{m}\right)$ and the muscle structure around this center. When muscular moving, original muscle point position which is $\left(x_{m}, y_{m}\right)$ is displaced, and muscle structure around it is also changed. So, for different face samples, these subparameters are not fixed value. Therefore, PRNet used to build 3D face is difficult to resolve $d_{m}$ and $\alpha_{m}$ exactly; and the extent of muscle movement in frontalization face is more weakened than that of real front face.

But the parameter of muscle moving direction $\theta_{m}$ can be estimated exactly, and 3D face could show muscle moving direction clearly. For the same area (e.g., No. $m$ ) in the different face sample, although its center $\left(x_{m}, y_{m}\right)$ in each sample may be a little displacement, the subparameters of $\theta_{m}$, including $p_{m, \theta, 1}\left(x_{m}, y_{m}\right), p_{m, \theta, 2}\left(x_{m}, y_{m}\right), \ldots, p_{m, \theta, n \theta}$ $\left(x_{m}, y_{m}\right)$, are only relative to the direction of muscle cells contraction and relaxation, while these cells around No. $m$ area have almost the same moving direction.

This can be proved through the theory of muscular structure and cells anatomy. The muscle on the face is striated muscle that is used for control expression [32, 33]. 
The shape of every muscle cell is slender; these cells array as bundle structure for a muscle, as shown in Figure 11. These cell contract and relax on one fixed direction, $\theta$ and $\pi+\theta$.

For every muscle, it is composed of a whole regular cells bundle, and its cells are contracting and relaxing in the same direction. So, for one of small area (No. $m$ ) in the cells bundle, it has the same moving direction as that of the whole bundle. Therefore, the area center in the $\theta_{m}$ resolving function (6) can be substituted by average center $\left(\bar{x}_{m}, \bar{y}_{m}\right)$ of this muscle bundle. So $\theta_{m}$ resolving function can be simplified as follows:

$$
\begin{aligned}
\theta_{m}= & F_{m u s, \theta, m}\left(\bar{I}_{x}, \bar{I}_{y}, p_{m, \theta, 1}\left(\bar{x}_{m}, \bar{y}_{m}\right), p_{m, \theta, 2}\left(\bar{x}_{m}, \bar{y}_{m}\right), \ldots,\right. \\
& \left.\cdot p_{m, \theta, n \theta}\left(\bar{x}_{m}, \bar{y}_{m}\right)\right) .
\end{aligned}
$$

Although No. $m$ area center may be displaced with the movement of muscle bundle, the subparameters $\left(p_{m, \theta, 1}\left(\bar{x}_{m}, \bar{y}_{m}\right), p_{m, \theta, 2}\left(\bar{x}_{m}, \bar{y}_{m}\right), \ldots, p_{m, \theta, n \theta}\left(\bar{x}_{m}, \bar{y}_{m}\right)\right)$ of $\theta_{m}$ are of fixed values; and the total number of subparameters in function (7) for all small areas is $n_{M} \times n_{\theta}$. Meanwhile, for the PRNet used to build face muscle model, its training samples number $n_{s}$ is very large and $n_{s} \gg n_{M} \times n_{\theta}$; and, according the theory of " $N: q$ rule" of parameter estimates [26, 27], subparameters for $\theta_{m}$ can be resolved. All the small areas moving direction $\left(\theta_{i}, i=1, \ldots, n_{\theta}\right)$ in the muscle model can be resolved effectively by PRNet. Although the muscle moving extended in the model is more weakened than that of the real one, they can still be strengthened according to their moving direction.

In the next section, in order to extract muscle moving direction from 3D face, face contour model is proposed and Fréchet distance is introduced to analyze contour lines; and muscle movement strengthening model is also designed to strengthen muscle movement following the moving direction. The muscle strengthening result is shown in Figure 12; "sad" can be intensified, especially on the brows, eyes, and mouth.

2.2. The Design of Facial Expression Strengthening Model. In order to strengthen muscle movement on the PRNet 3D face to intensify expression features, our expression strengthening model is composed of two parts: face $3 \mathrm{D}$ contour model for muscle moving parameters extracting (especially direction $\theta$ ) and face muscle movement model for expression strengthening.

2.2.1. Face Contour Model for Extracting Muscle Moving Direction. Extracting the muscle moving direction for every small face area is the core for enlarging muscle movement and strengthening facial expression. In this paper, $3 \mathrm{D}$ contour model and Fréchet distance are introduced to extract muscle moving direction.

The contour model is designed based on the $3 \mathrm{D}$ face. In the case of muscular contraction and relaxation, the depths

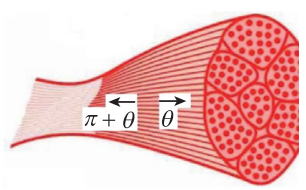

FIgURE 11: The slender muscular cell and its reticular structure.

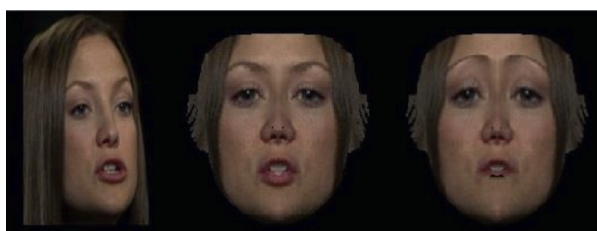

(a)

(b)

(c)

Figure 12: Sad face (a), its frontalization (b), and the expression strengthening result (c).

(the thickness of muscle and bone) of different face areas are changed, and the contours shape around muscle would be changed significantly. As shown in Figure 13, the contours with the same depth are colored with the same color and it can be shown clearly that contours shapes of happy face are much different from that of neutral face, especially the contours on the eyes, cheeks, and mouth.

In order to simplify the contour model for computer algorithm calculation, the 3D face is divided into 7 contour layers with different depth; and, in the head horizonal direction, 3D face was divided into four expression sensitive parts along skin: forehead part, brow and eye part, cheek part, and moth part. So, there are a total of 28 parts in the half face; and 28 muscle areas would be extracted from these parts to strengthen muscle movement. The whole expression strengthened frontalization face can be gotten by combining this strength half face with its mirror.

For each small part, the place in which Fréchet distance is the maximum between expression face contours and standard neutral face contours is the most significant muscle movement area in this part. Fréchet distance is a very useful method to analyze the distance between two curves. Its direction is muscle moving direction, and length is in ratio with real muscular movement area extent, as shown in Figure 14.

Given two contours curves $E, S$, their Fréchet distance $F(E, S)$ is [33]

$$
\begin{aligned}
F(E, S) & =\inf _{\alpha, \beta} \max _{t \in[0,1]}\{d(E(\alpha(t)), S(\beta(t)))\}, \\
D & =\left(\begin{array}{ccccc}
d_{1,1} & \ldots & d_{1, k} & \ldots & d_{1, n s} \\
\vdots & & \vdots & & \vdots \\
d_{i, 1} & \ldots & d_{i, k} & \ldots & d_{i, n s} \\
\vdots & & \vdots & & \vdots \\
d_{n e, 1} & \ldots & d_{n e, k} & \ldots & d_{n e, n s}
\end{array}\right), \\
d_{i, k} & =\sqrt{\left(x_{i}-x_{k}\right)^{2}+\left(y_{i}-y_{k}\right)^{2}}
\end{aligned}
$$




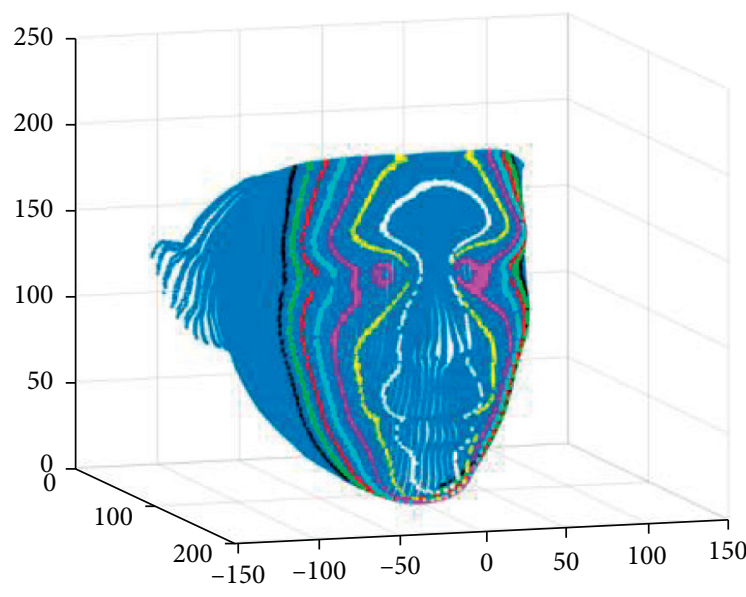

(a)

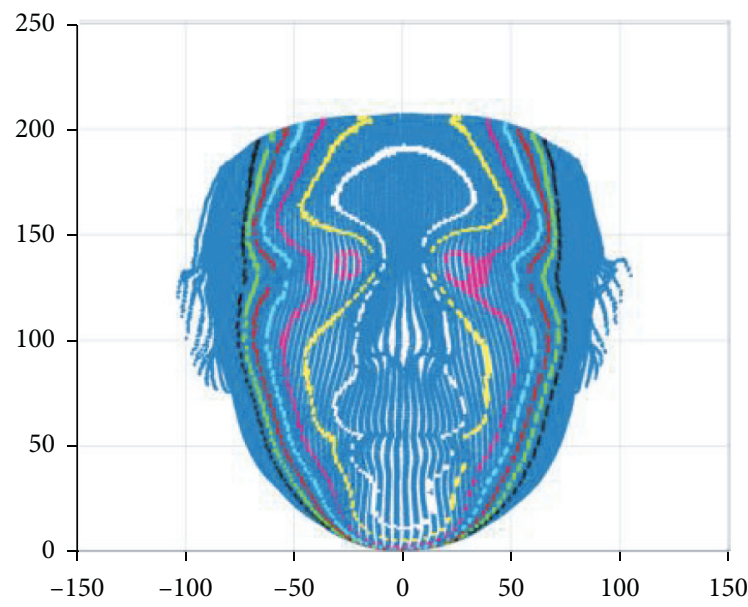

(c)

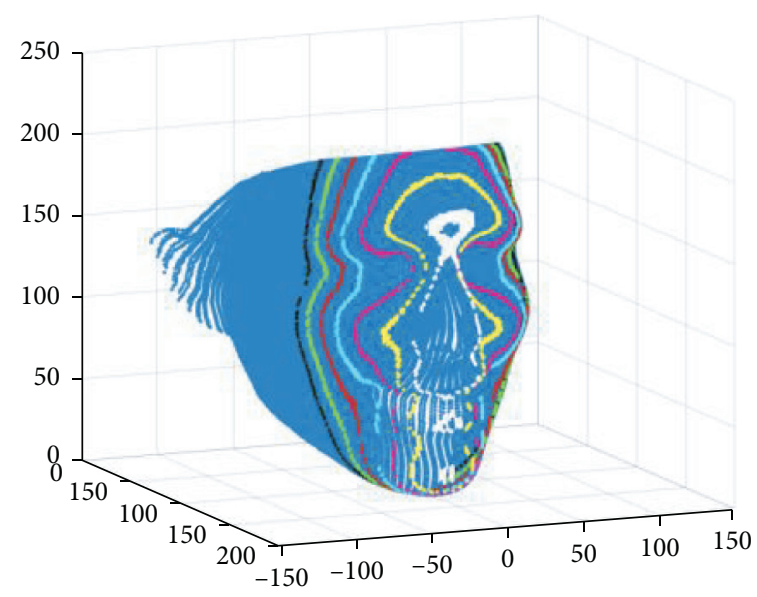

(b)

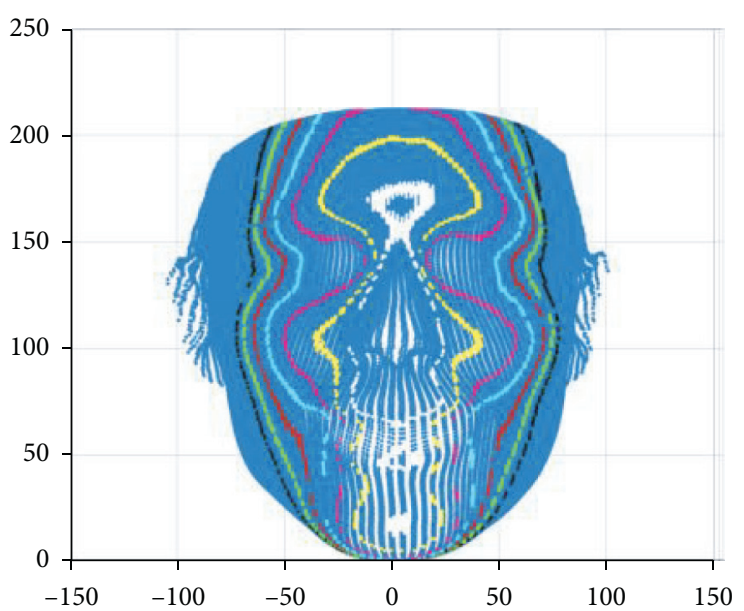

(d)

Figure 13: The contours (color lines) difference between happy and neutral face. (a) 3D neutral face, (b) 3D happy face, (c) neutral face frontalization, and (d) happy face frontalization.

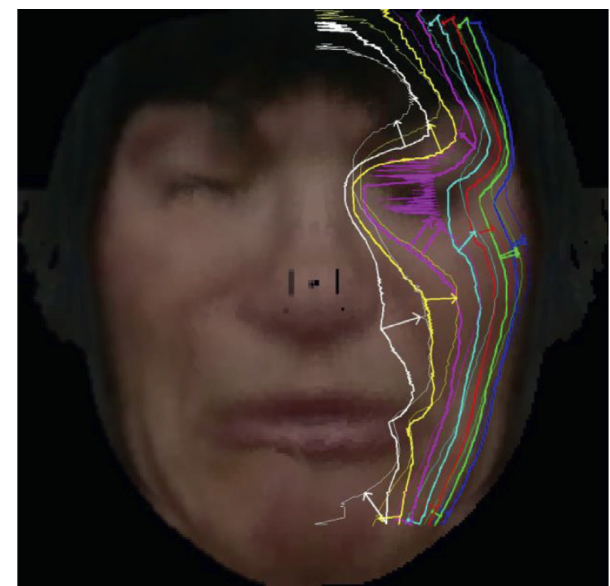

Figure 14: The Fréchet distances (color arrows) and muscle moving direction.

where $\left(x_{i}, y_{i}\right)$ and $\left(x_{k}, y_{k}\right)$ are the points on these two contours and $n e, n s$ are the points number. $\alpha, \beta$ are a kind of distance measure and can be shown through distance matrix
$D$ which is the combination of connecting lines between two contours.

Through processing matrix $D$, a group of connect line distance $d_{1}, d_{2}, \ldots, d_{n d}$ could be extracted as thresholds can meet the requirement of curve distance. $F(E, S)$ is shortest and is the infimum of $d_{1}, d_{2}, \ldots, d_{n d}$ denoted as "inf" in function (9). Fréchet distance vector is shown as the color arrows in Figure 14. And the muscle movement parameters in each of face parts, including muscle area center $\left(x_{m}, y_{m}\right)$ and moving direction $\theta$, can be gotten.

As nonfrontal face is frontalized just through the half face toward camera, the muscle is only strengthened on this half face, and then its mirror is made to build whole front face.

2.2.2. Muscle Movement Strengthening Model. 3D Gauss model is very convenient for controlling muscle contraction and relaxation in different small face area. And this model could combine these muscle movements in adjacent areas together to strengthen the whole face expression. 
This Gauss model can simulate muscle movement by inclining its peak and enlarging its zone extent and its projection on the $X Y$ plane much similar to the muscle movement, as shown in Figures 15(a) and 15(b). In order to simplify the muscular model, the inclination angle $\alpha$ is set to minimum to make the strengthening effect maximum. The adjustable parameter is the area extent $d$ of muscle movement; it could be enlarged following the direction $\theta$ to enlarge muscle movement area and strengthen muscle movement.

As frontalization face is the projection of $3 \mathrm{D}$ face on the $X Y$ plane, the muscle strengthening process can be simplified by calculating the projection of the 3D Gauss model on $X Y$ plane, as shown in function (10). This function could give every point on the face image a new position $\left(X_{M, i}, Y_{M, i}\right)$ to change face shape and intensify facial muscle contraction or relaxation, for the No. $i$ area:

$$
\left\{\begin{array}{l}
X_{M}=X+X_{H, i} \cdot \exp \left(-\frac{\left(X-x_{m, i}\right)^{2}}{2 d_{i}^{2}}\right) \sin \left(\pi+\theta_{i}\right), \\
Y_{M}=Y+Y_{H, i} \cdot \exp \left(-\frac{\left(Y-y_{m, i}\right)^{2}}{2 d_{i}^{2}}\right) \cos \left(\pi+\theta_{i}\right),
\end{array}\right.
$$

where $(X, Y)$ is the original coordinate of face point, $\theta$ is the muscle moving direction, and $X_{H}, Y_{H}$ are related to the model inclination angle $\alpha$ which have been set to make muscle movement maximum.

The moving direction $\theta$ in function (10) is embodied as $\sin (\pi+\theta)$ and $\cos (\pi+\theta)$. It can control the contraction or relaxation area rotating to the muscular moving direction on the $X Y$ platform.

This function could not only make muscular contract and relax but also make this strengthening area connect with its surround areas smoothly. For example, in the $X$ direction, it gives different position with different coordinate displacement $X_{H} \cdot \exp \left(-\left(\left(X-X_{M, 0}\right)^{2} /\left(2 d^{2}\right)\right)\right)$ $\sin (\pi+\theta)$. Following the $3 \sigma$ principle of Gauss model, the boundary of real area to strengthen muscle movement is set as $\pm 2 \sigma$ in our method, which is double of muscle movement area extent $d(\sigma=d)$, and the coordinate is $\left[X_{M, 0}-2 d\right.$, $X_{M, 0}+2 d$ ], where the muscle movement (decreasing to $5 \%)$ is close to 0 on the area boundary. In the center of this area, the displacement value is maximum: $X_{H} \sin (\pi+\theta)$. For the points on the two sides, their coordinate displacement is decreased gradually to the area boundary and can connect with the adjacent areas smoothly, as shown in Figure 15(b). The same relationship also suits the $Y$-axis and its muscle movement model $Y_{H} \cdot \exp \left(-\left(\left(Y-Y_{M, 0}\right)^{2}\right.\right.$ $\left.\left./\left(2 d^{2}\right)\right)\right) \cos (\pi+\theta)+Y$.

This function can make the muscles of some areas partially overlap each other to make whole face muscle moving. For the point in the overlap areas, their coordinate functions are designed as follows:

$$
\left\{\begin{array}{l}
X_{M}=X+\sum_{i=1}^{n_{m}} X_{H, i} \cdot \exp \left(-\frac{\left(X-x_{m, i}\right)^{2}}{2 d_{i}^{2}}\right) \sin \left(\pi+\theta_{i}\right), \\
Y_{M}=Y+\sum_{i=1}^{n_{m}} Y_{H, i} \cdot \exp \left(-\frac{\left(Y-y_{m, i}\right)^{2}}{2 d_{i}^{2}}\right) \cos \left(\pi+\theta_{i}\right) .
\end{array}\right.
$$

The muscular contraction or relaxation for multioverlap areas is shown in Figure 16(c); this is a "sad" image. And the expression strengthening face is shown in Figure 16(d); the shapes of brow, eye, cheek, and mouth are changed significantly to strengthen "sad" compared to original frontalization face (Figure 16(b)).

\section{Experiment Results}

For testing this expression recognition method effectively, the library SFEW2.0 is used. This library concludes a great deal of photos taken from many famous films; human faces in these photos are taken in the wild and their heads poses are varied. The common FER methods might be disturbed seriously by this library, and recognition rates of traditional methods are only 60 70\% in the wild. For example, MEGVII Face++ is a famous face image processing company; its expression recognition software also makes a lot of mistakes for the images in SFEW2.0 library. While the face frontalization and expression strengthening method presented in this paper can solve these problems, these faces, the expression of which cannot be recognized exactly by Face++, can also be recognized exactly after face frontalization and expression strengthening.

The following are the experiment results with different face poses and different expressions. Face++ software failed to detect expression from these original faces. Meanwhile, after our face frontalization, some expressions can be recognized, and, after expression strengthening, the facial features on the strengthened frontalization face are more significant than those of original frontalization face, and recognition result is exact. The FER accuracy for different expressions increases by $10 \%$ in average.

Figure 17 shows the expression recognition result of rising head. According to the library label, this man is "sad." Meanwhile, as his head is rising, the sad features are all dislocated. So this expression is mistakenly identified as "neutral" by Face++ platform, as shown in Figure 18.

After face frontalization, these sad features are readjusted and are similar to those of front face, as shown in Figure 17(b). Through this image, Face++ can rightly identify expression as "sad," as shown in Figure 19; the prediction confidence for "sad" is $41.35 \%$ and is larger than those in the other expression kinds.

After expression strengthening, the features of "sad" on cheeks and brows on the frontalization face are strengthened automatically. And Face++ can recognize the "sad" much 


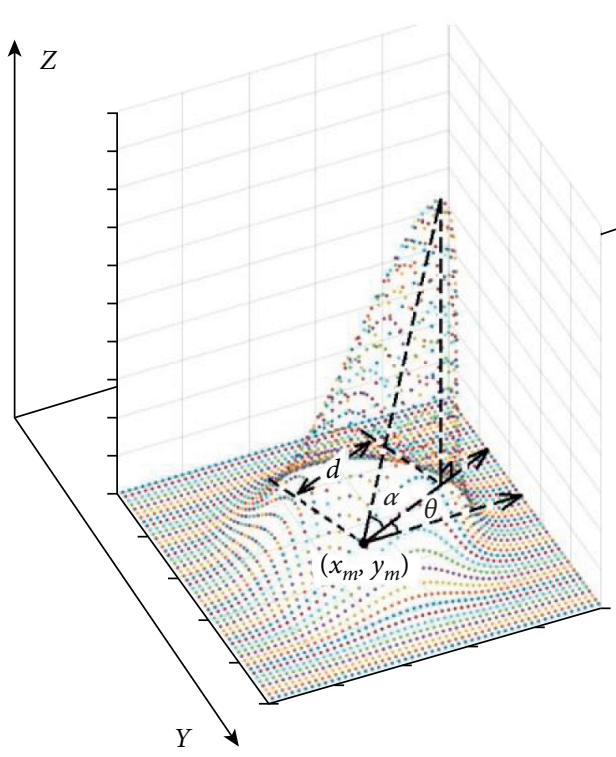

(a)

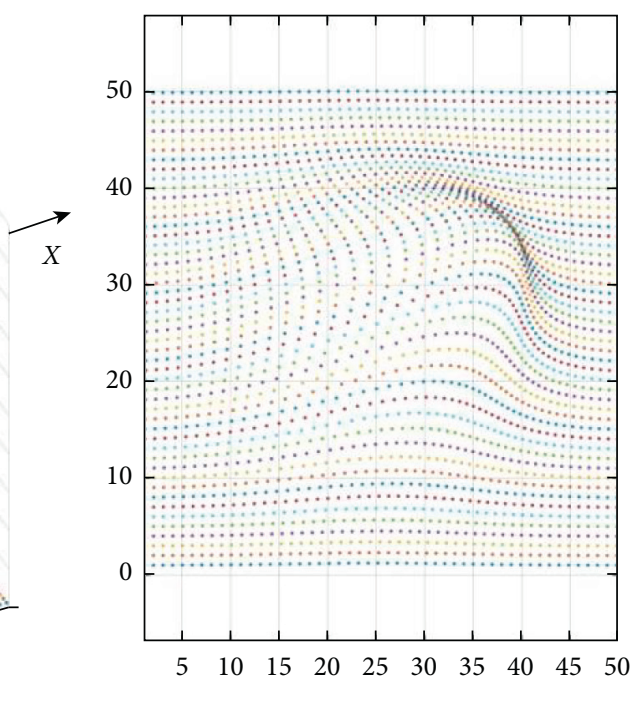

(b)

Figure 15: Inclined 3D Gauss model (a) and its projection on XY plane (b).

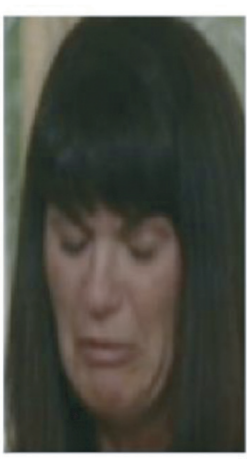

(a)

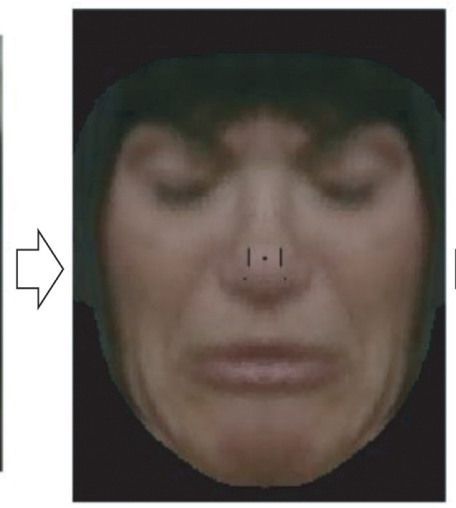

(b)

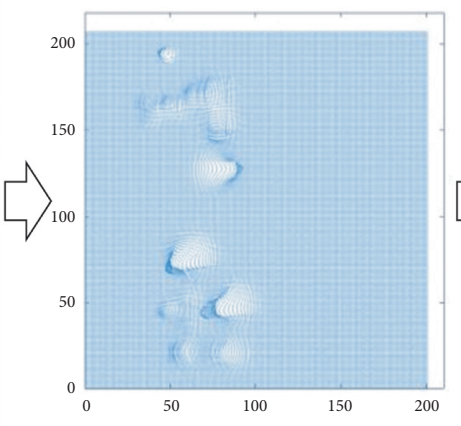

(c)

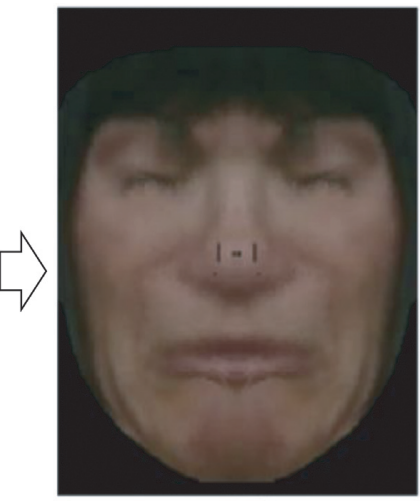

(d)

Figure 16: The whole facial expression strengthening. (a) Original face, (b) its frontalization, (c) muscular contraction and relaxation through function (9), and (d) "sad" expression strengthening result.

exactly; the prediction confidence increased from $41.35 \%$ to $68.55 \%$, as shown in Figure 20.

In Figure 18, there are 7 horizontal bars on the right of Face++ platform to show the prediction confidence of the seven expressions. From top to bottom, the seven expressions are "happy," "neutral," "surprise," "sad," "disgust," "angry," and "fear," and these are noted in English as shown in Figure 18. The most probable expression in the recognition result is shown as orange bar.

Figure 21 shows the expression recognition result of turning head. According to the library label, this man is smiling. Meanwhile, as his head is turning and bowing and he is closing his eyes, the features in the original face are very blurly in the dim light. This expression is mistakenly identified as "sad" by Face++ platform, as shown in Figure 22.

After face frontalization, although the front face is gotten, the expression features are insignificant and Face++ is introduced to mistakenly identify as "disgust," as shown in Figure 23.

After expression strengthening, the features of smile are intensified on this frontalization face automatically, especially the features on the cheek; the cheek muscle is squeezed into two sides to make the smile more 

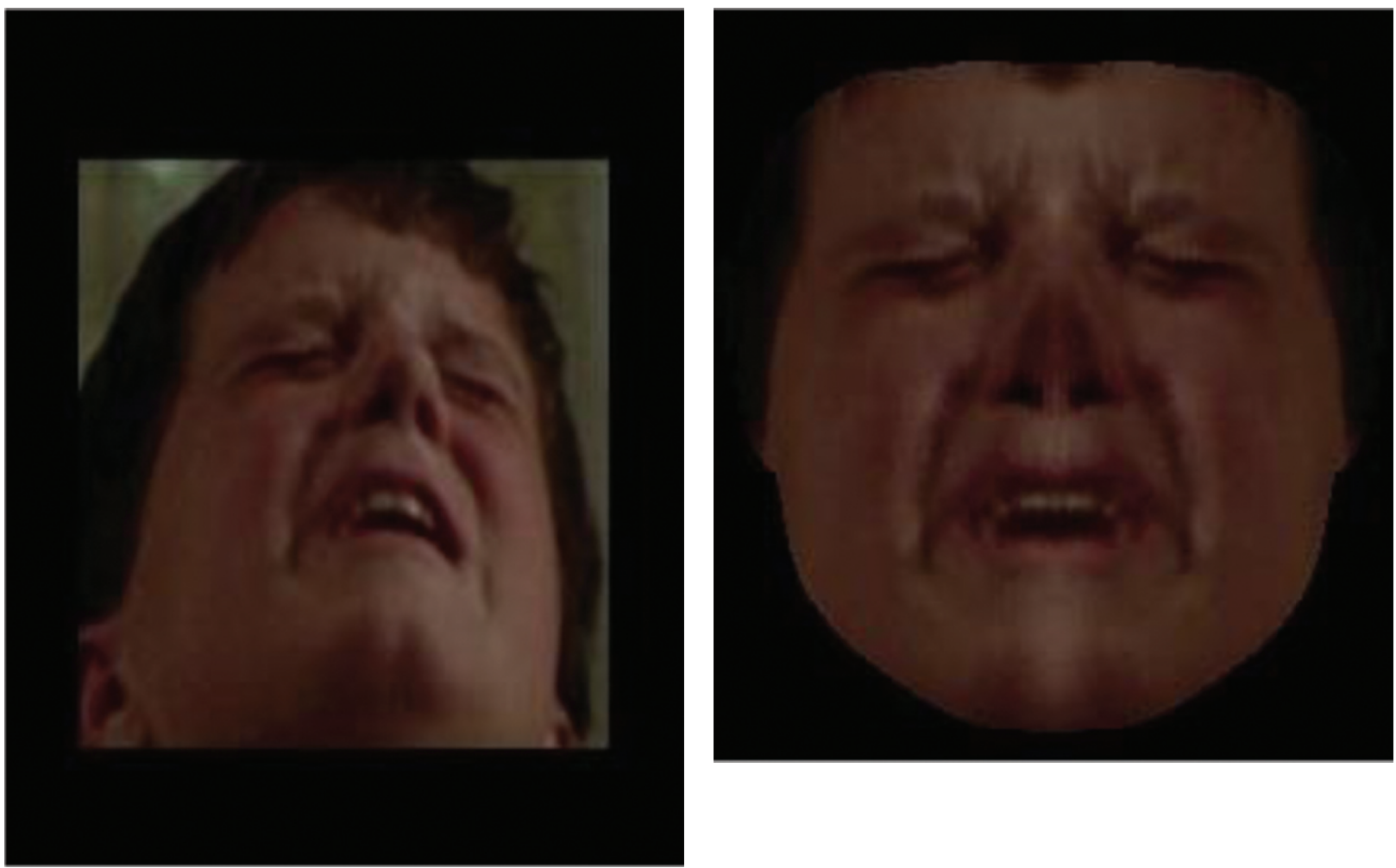

(a)

(b)

FIgURE 17: Continued.

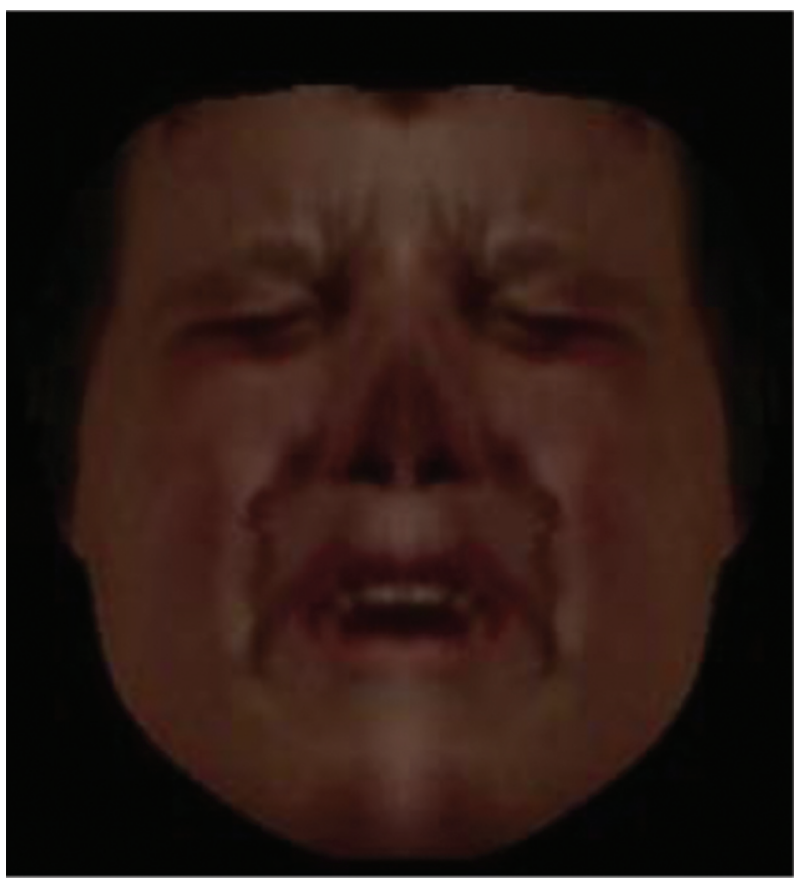

(c)

FIgURe 17: Sad face (a), its frontalization (b), and expression strengthening result (c).

significant. Through this strengthening image, Face ++ can recognized the "smile" more exactly, as shown in Figure 24 , and prediction confidence is close to $80 \%$.
Figure 25 shows the expression recognition result of turning head to left. According to the library label, the human is "angry." Meanwhile, as his head is turning, this 


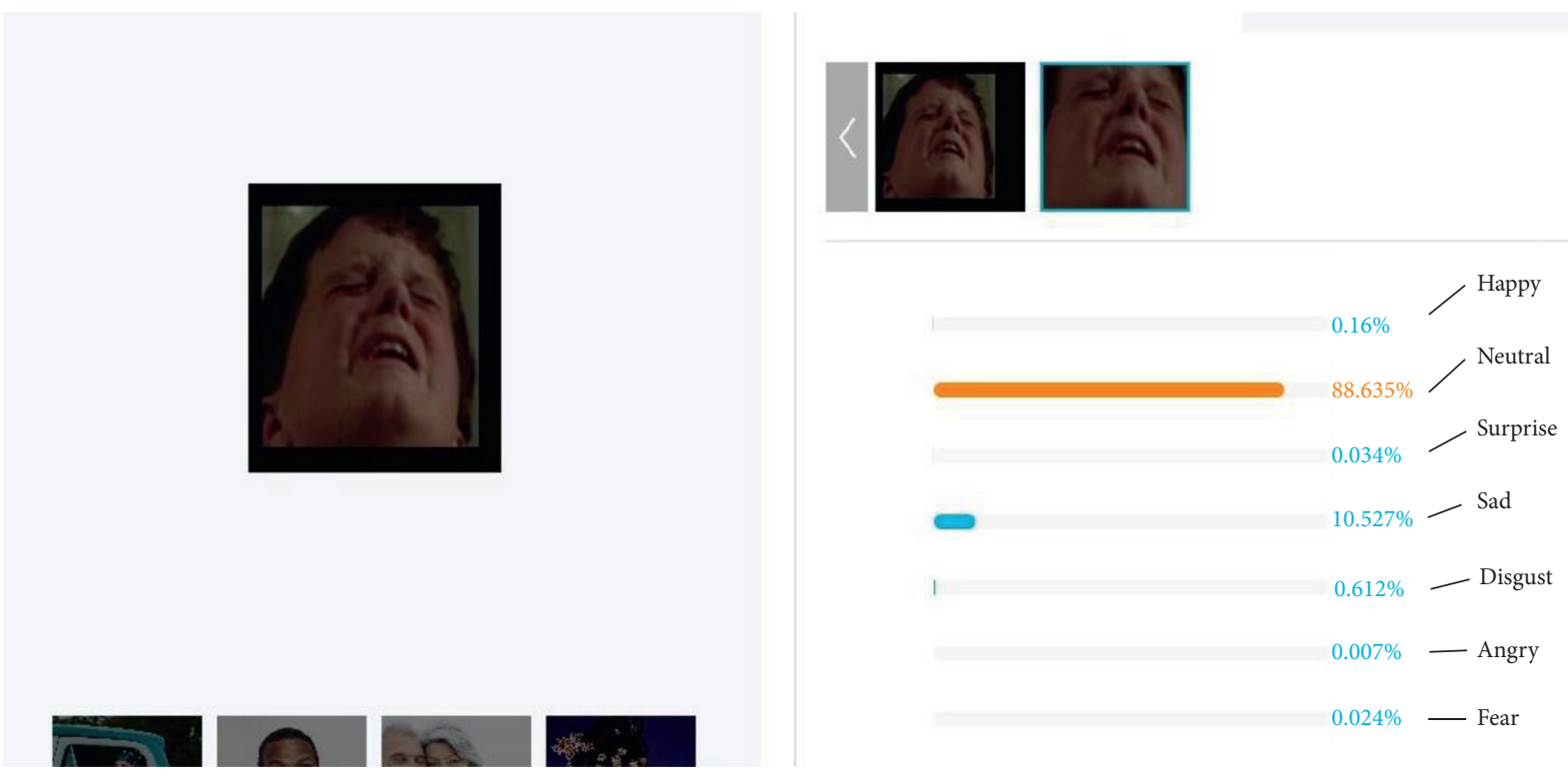

FIGURE 18: Original sad face is mistakenly recognized as "neutral."
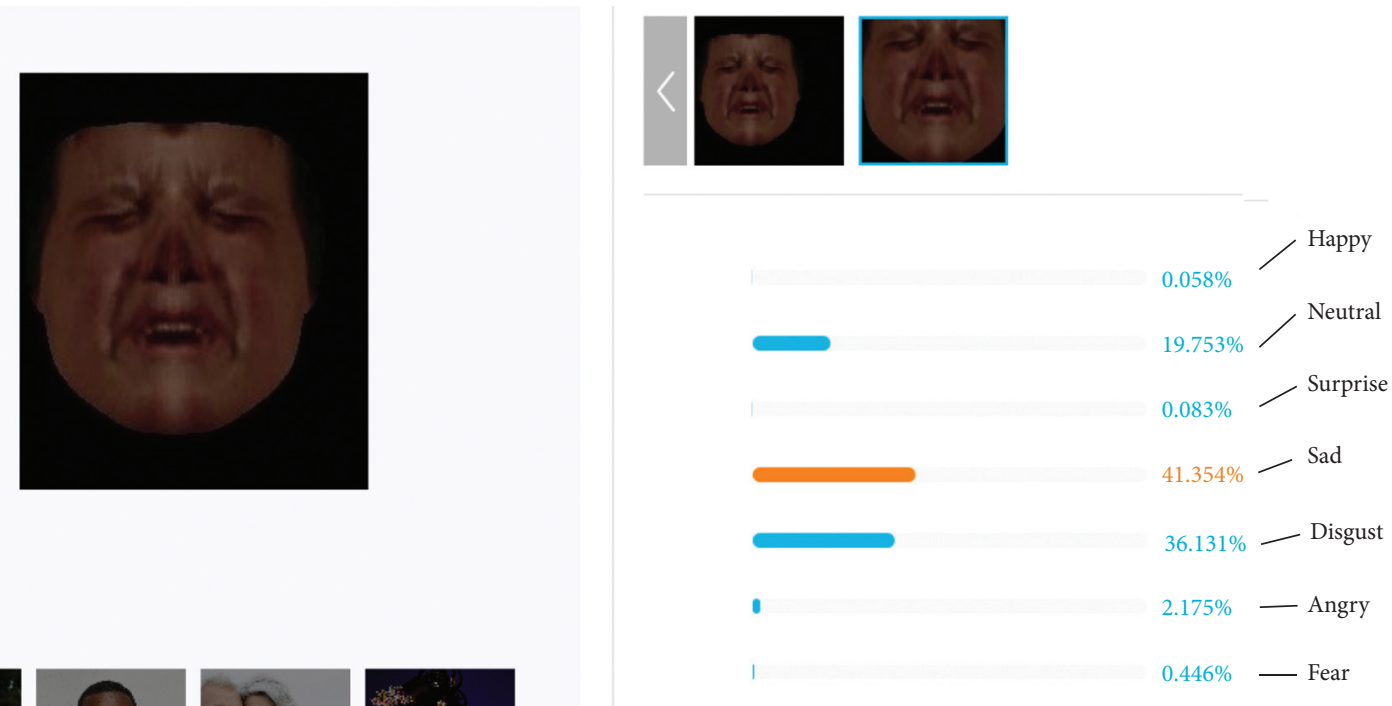

FIGURE 19: The frontalization face is recognized as "sad" but prediction confidence is only $41.35 \%$.
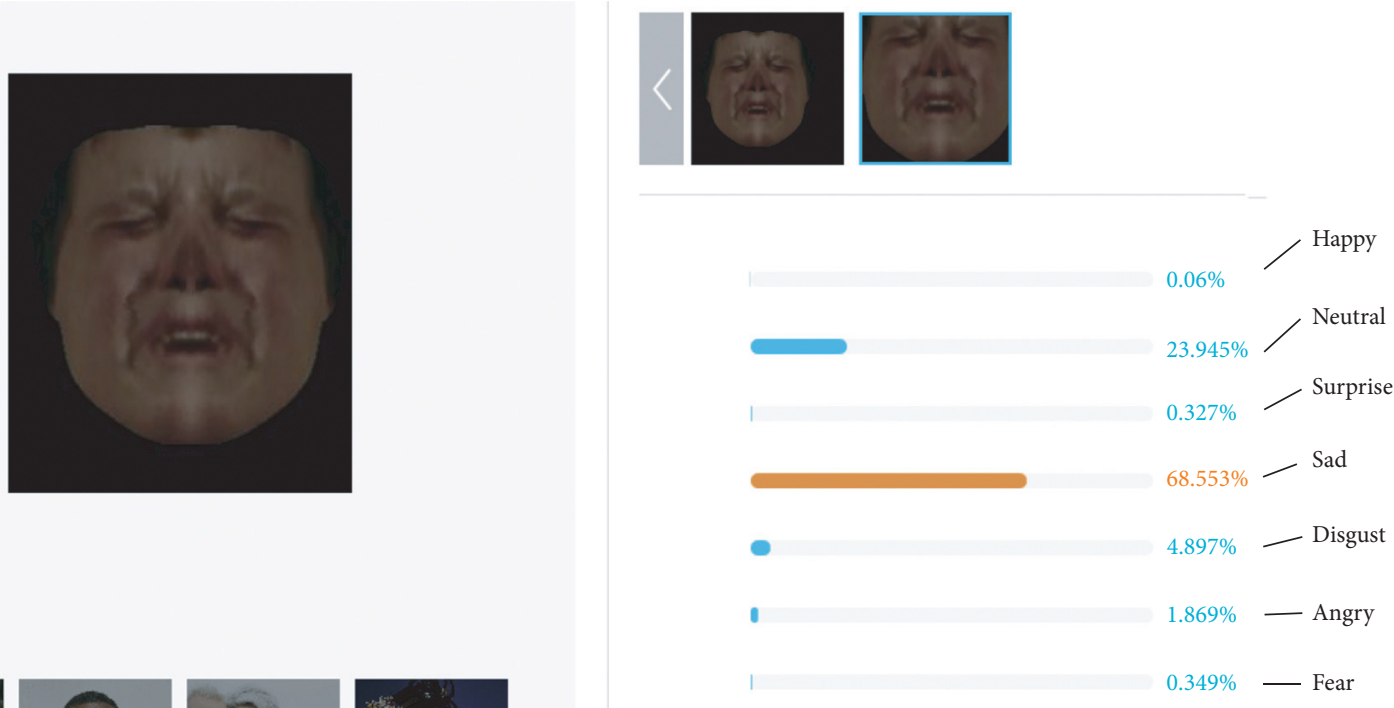

FIGURE 20: The expression strengthened face is correctly recognized as "sad" and prediction confidence increases to $68.55 \%$. 


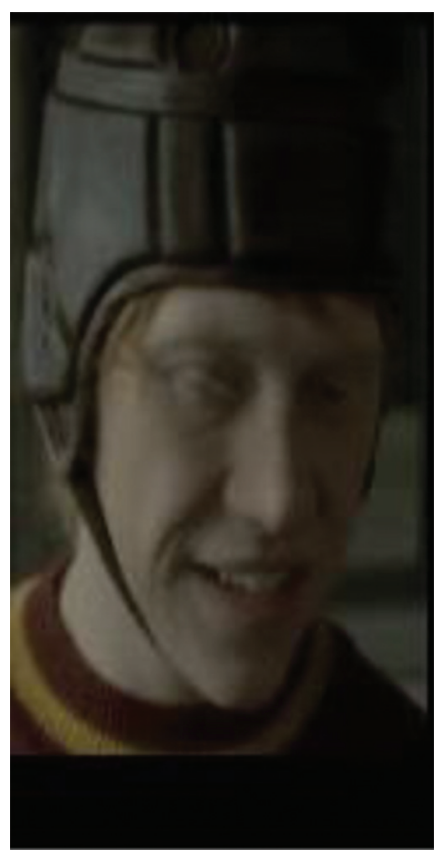

(a)

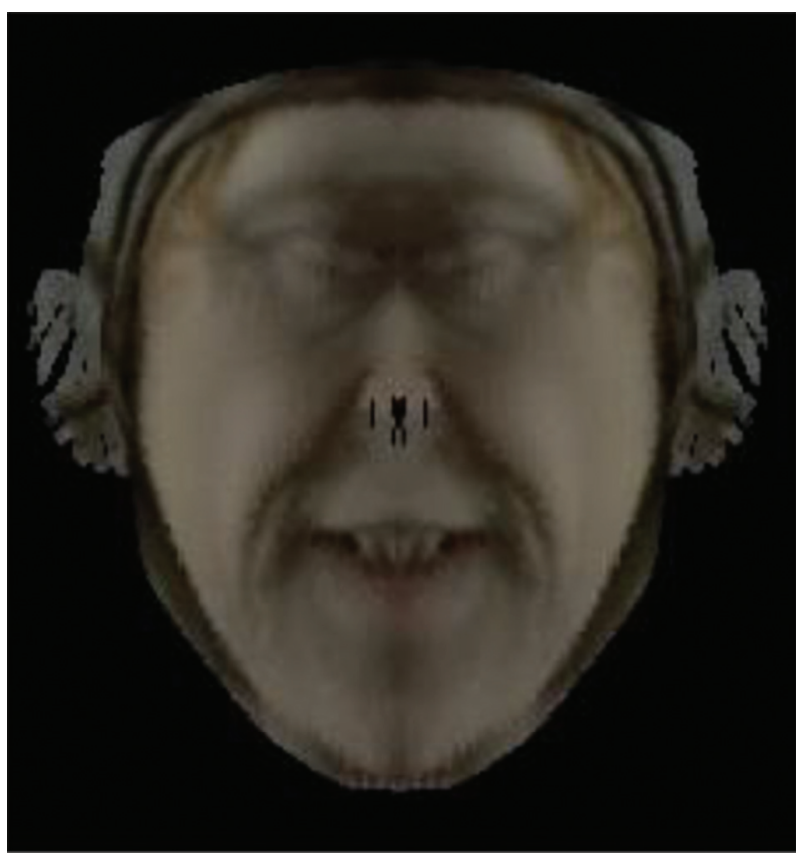

(b)

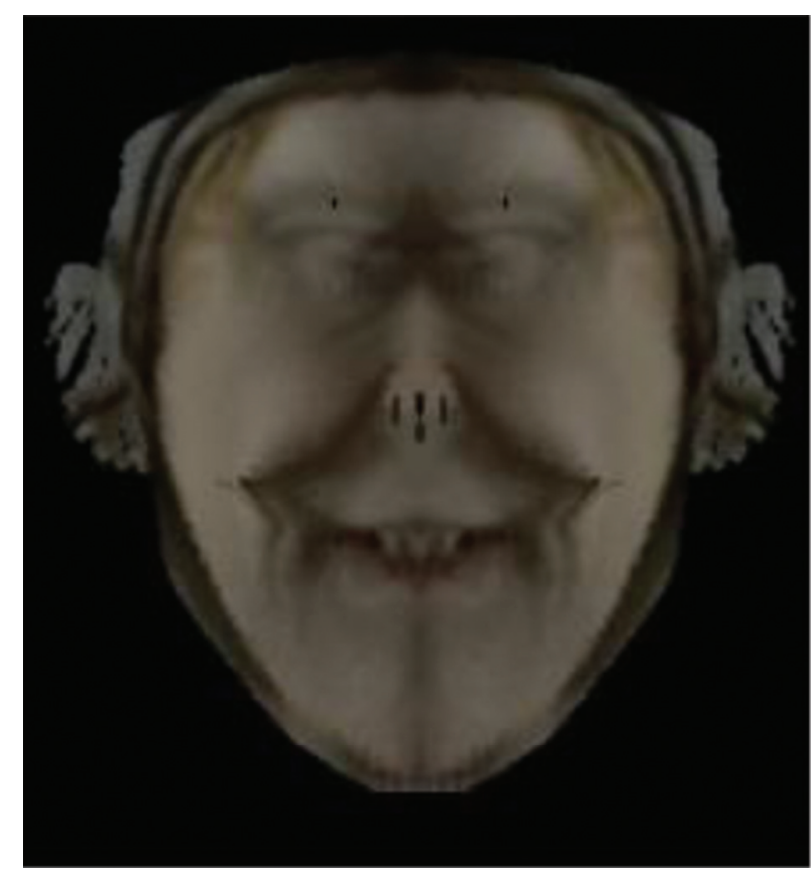

(c)

Figure 21: Happy face (a), its frontalization (b), and expression strengthening result (c).

expression is mistakenly identified as "surprise" (prediction confidence $47.16 \%$ ) and "neutral" (prediction confidence $46.68 \%$ ) by Face++, as shown in Figure 26.

For the frontalization face, it is recognized as "sad," which is a little close to "anger" than "surprise," as shown in Figure 27.

After expression strengthening, the features of "sad" on brows and mouth on the strengthened frontalization face are more significant. And Face++ can recognize the "anger" more exactly; the prediction confident is $69.4 \%$, as shown in Figure 28.

\section{Results-Related Discussion}

For the SFEW database introduced to test our expression recognition method, it is a very difficult database for testing nonfrontal expression recognition. The reported recognition rates for database SFEW are often very less, because the facial expression features in the wild are often deformed and translated. For example, the deep learning method, Multiple deep CNNs, is 55.96\% [21], RAN (VGG16+ResNet18) is $56.4 \%$ [30], gACNN is $54.47 \%$ 


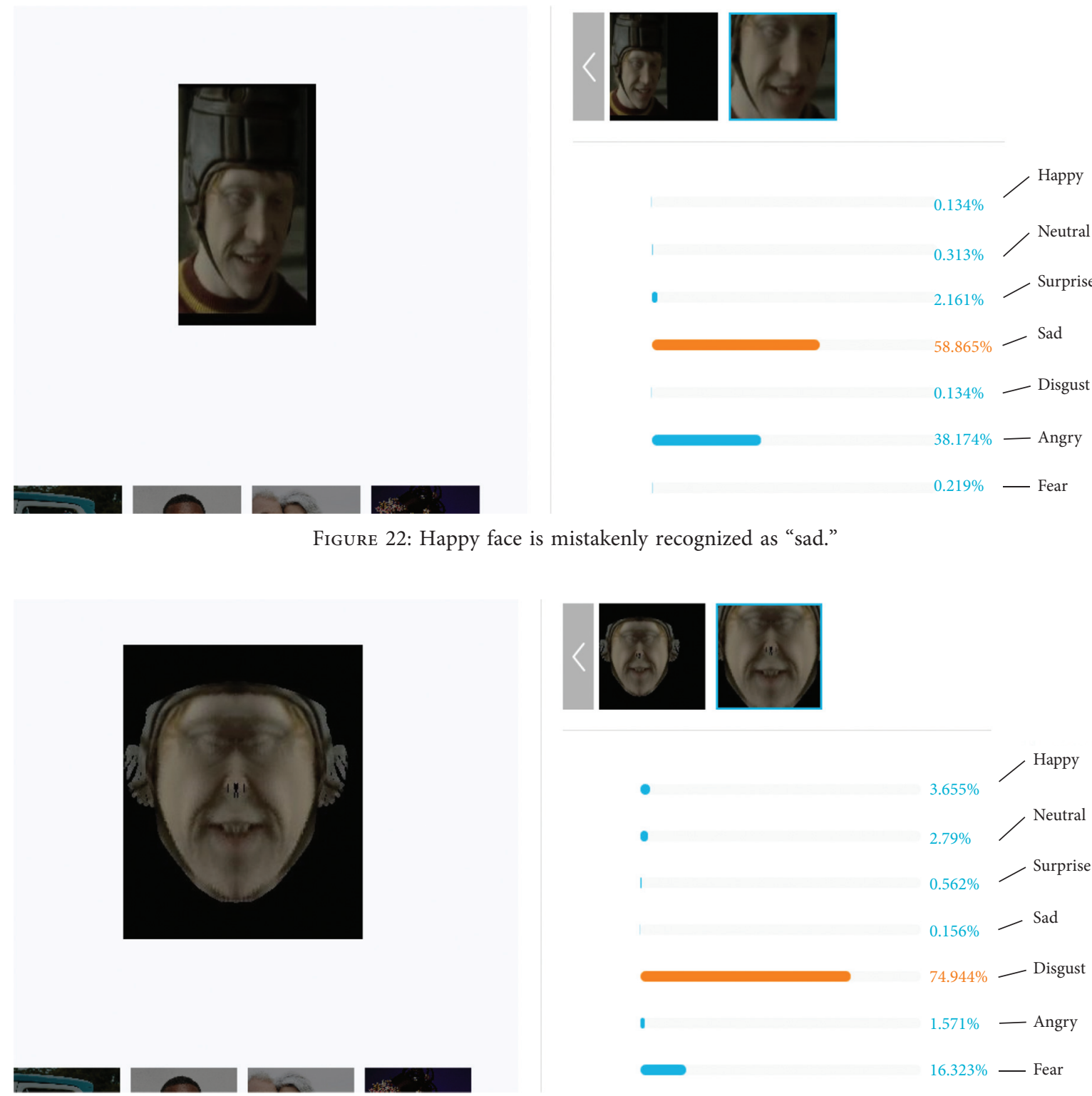

FIgURE 23: The frontalization face is mistakenly recognized as "disgust.".
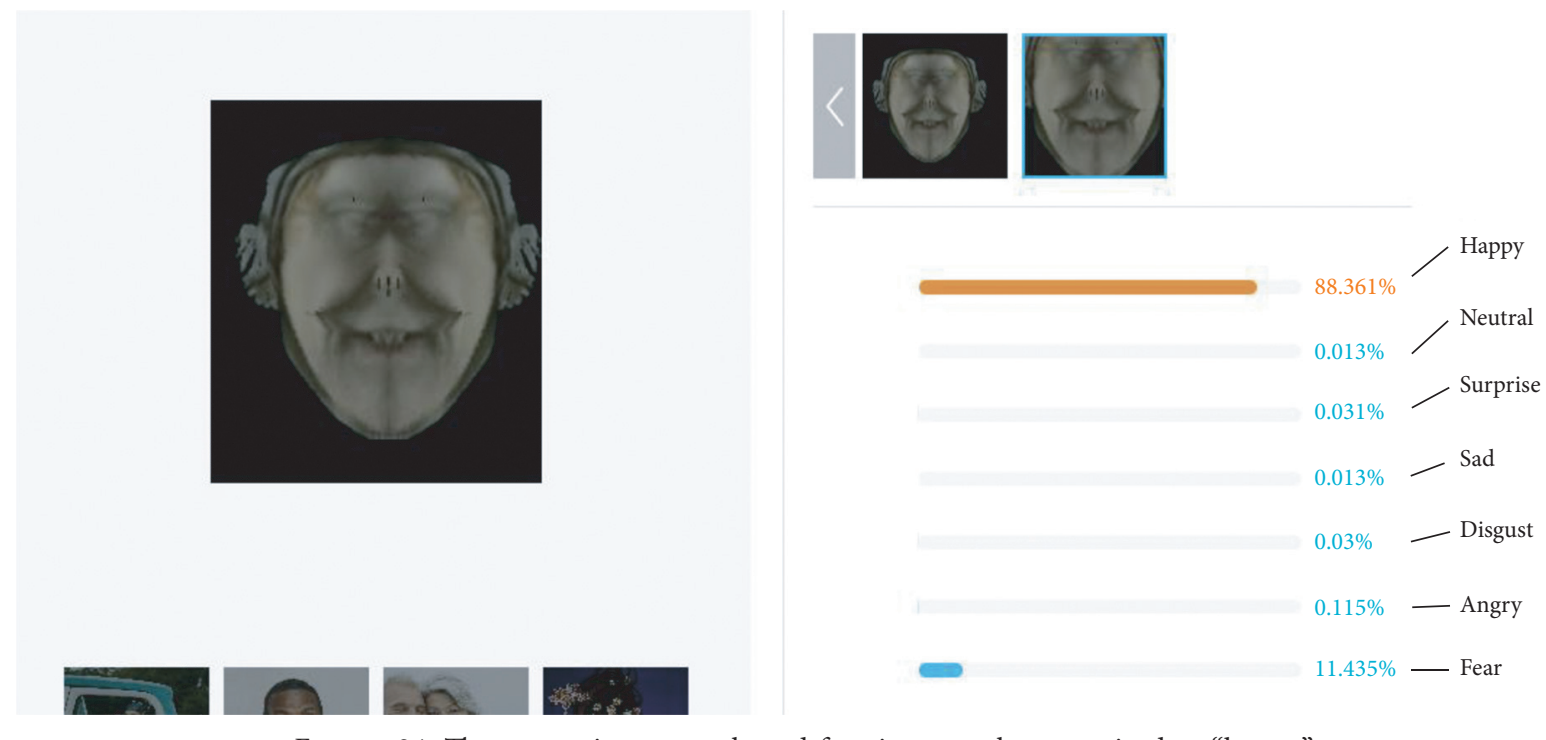

FIGURE 24: The expression strengthened face is correctly recognized as "happy" 


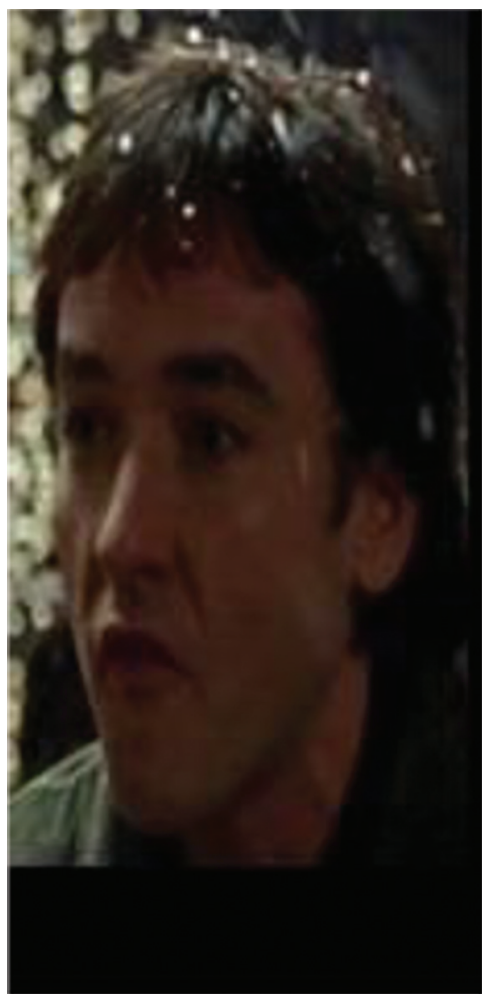

(a)

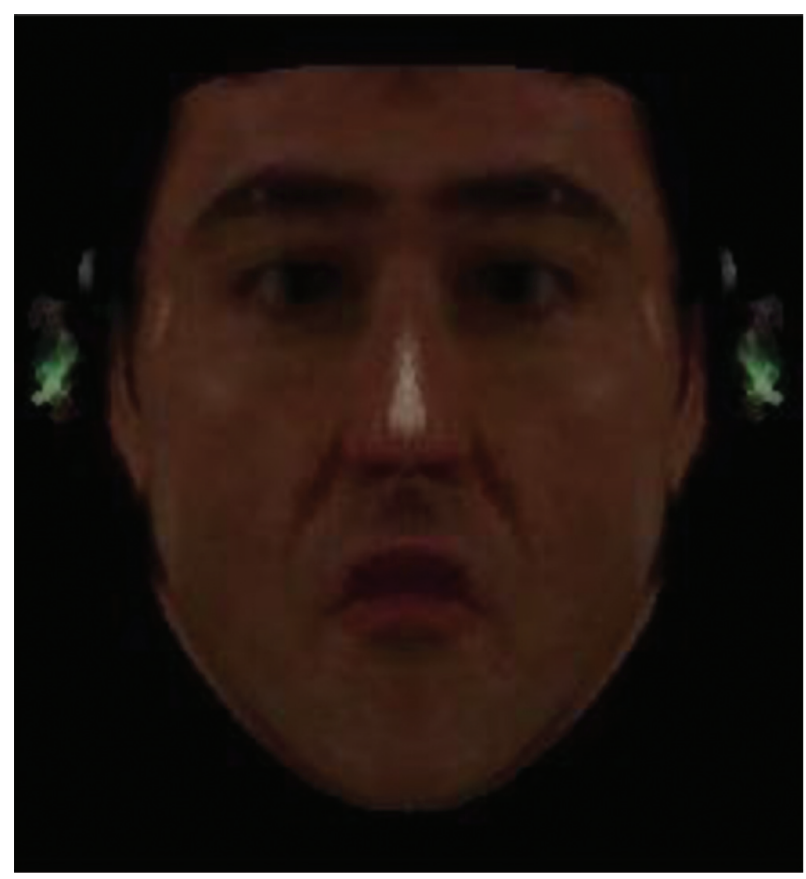

(b)

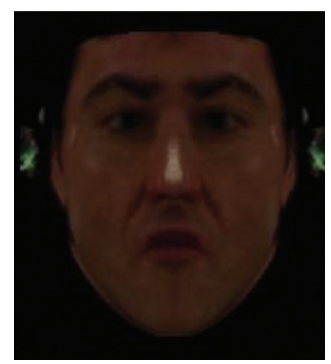

Figure 25: Angry face (a), its frontalization (b), and expression strengthening result (c).

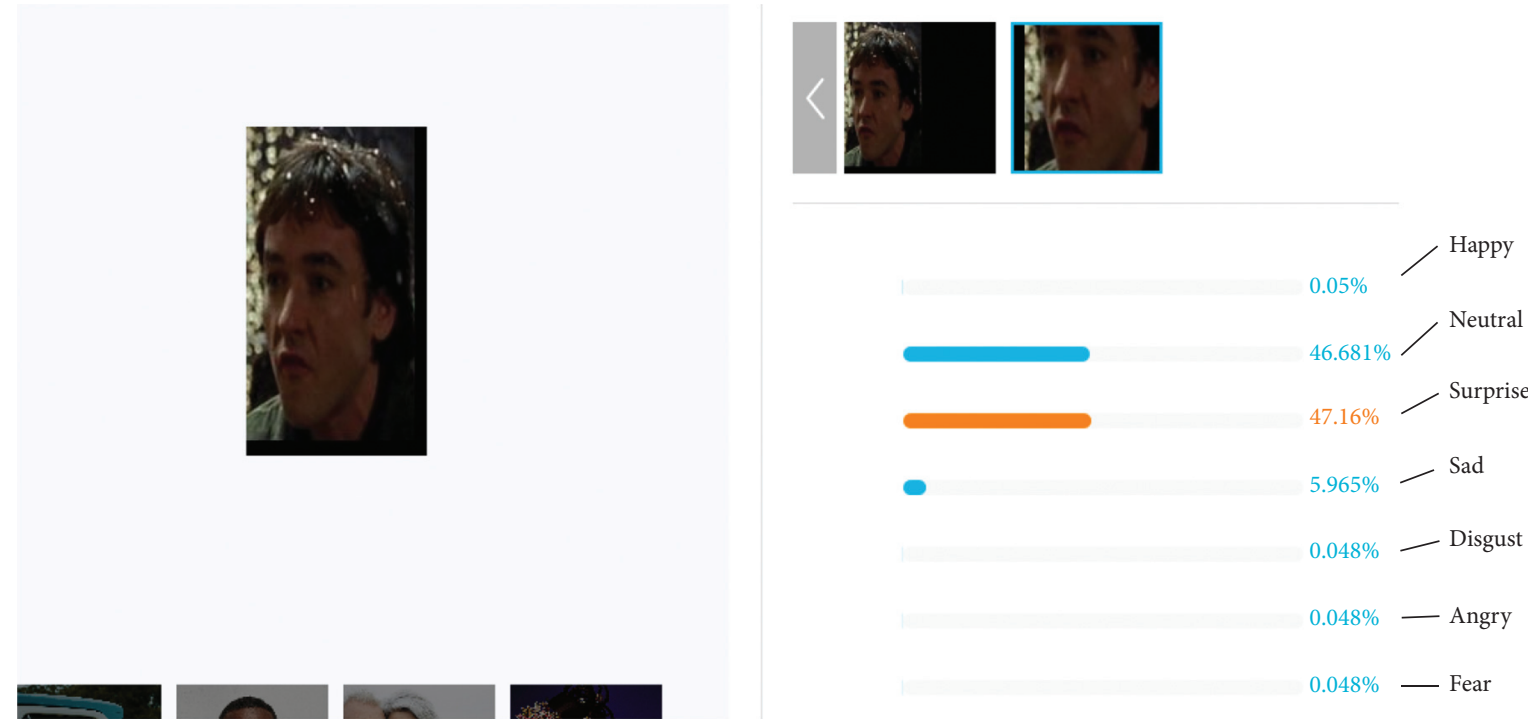

FIGURE 26: Angry face is mistakenly recognized as "neutral" and "surprise."

[22], and ensemble IL-CNN [24] is 59.41\%. Even for unpleasantness and pleasantness recognition, the recognition rates are still very low; for example, the reported recognition rates of ensemble IL-CNN [24] for unpleasantness and pleasantness are just $64.82 \%$ and $73.7 \%$.
Meanwhile, through face rebuilding method presented in this paper and muscle movement rebuilding and intensifying method, the expression on the nonfrontal face can be recognized more exactly from its rebuild front face, which can also strengthen muscle movement during this 


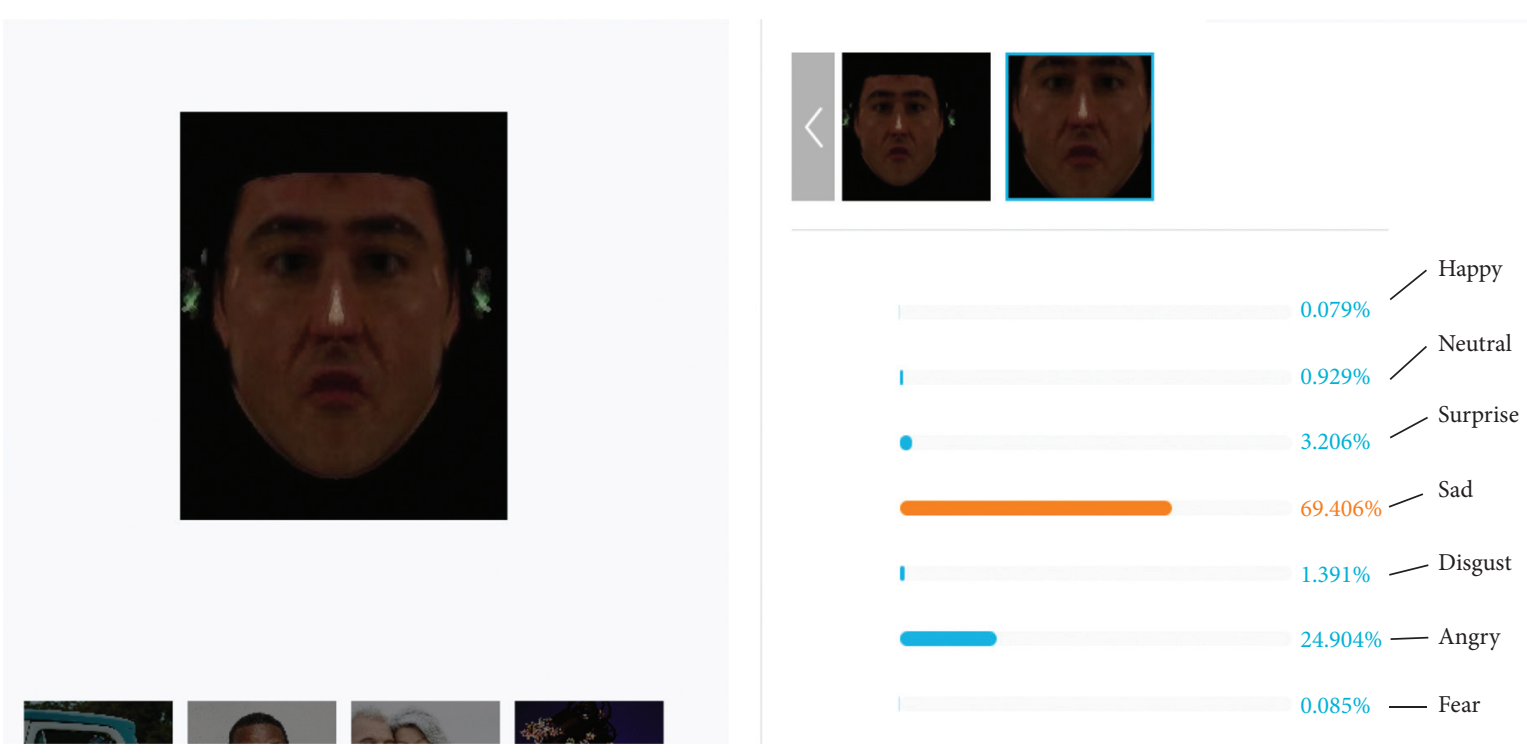

Figure 27: The frontalization face is recognized as "sad."
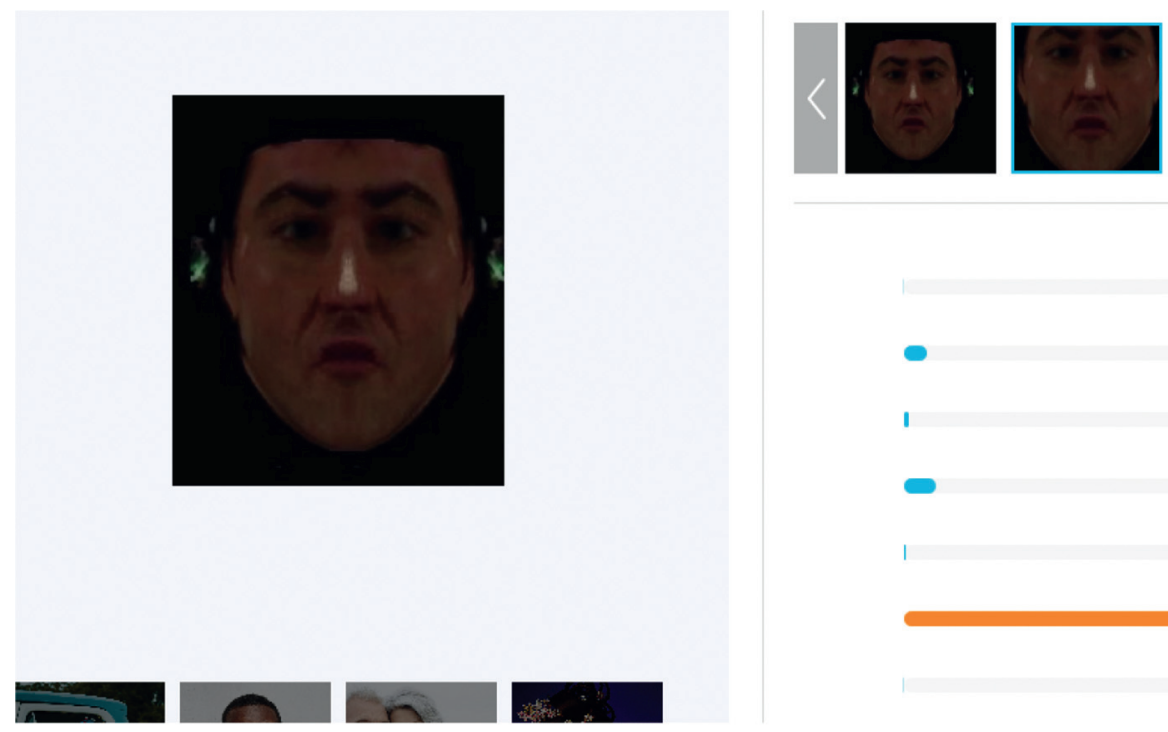

FIGURE 28: The expression strengthened face is correctly recognized as "anger."

frontalization process. And the expression rate for different expressions can be increased by $10 \%$ on average in the test.

\section{Conclusions}

Facial expression recognition (FER), especially in the wild, is a hotspot and also a challenge for AI. In order to solve the problem caused by face pose changing, this paper proposed a face frontalization and expression strengthening method for these nonfrontal face images. This method could build 3D face for each face in the wild and extract the front face through projection. And in order to improve FER accurate, this method has taken advantage of the features of face symmetry to build frontalization face. But the frontalization face cannot be used for FER directly, because, according to the theory of "N:q rule" of parameter estimates and the theory of muscle structure anatomy, this paper had proved that expression features in the frontalization face might be more weakened than those of real front face. And the parameters of muscular facial expression, except moving direction, cannot be estimated effectively. In order to strengthen muscle movement to highlight expression features, face contour model and muscle movement strengthening model are designed in this paper. Face contour model could extract the muscular moving direction in different face areas. Muscle movement strengthening model could intensify muscle movement in these areas. The expression strengthened frontalization face would be much 
suitable for expression recognition. The expression in nonfrontal faces, which cannot be recognized by the famous MEGVII Face++ software effectively, can be recognized exactly after face frontalization and expression feature strengthening.

\section{Data Availability}

The database used in this manuscript is SFEW2.0. It can be download from https://pan.baidu.com/s/ 1IktnrO0WTQoGNsX8cNSviQ, and download password is "ztum."

\section{Disclosure}

All the human faces, except Figures 2, 6, and 8 which had obscured their eyes, used in this manuscript are taken from the open database SFEW2.0. When this SFEW2.0 is built by its establishers, all the patients whose figures are sampled by this database have given consent for the clinical study to be published.

\section{Conflicts of Interest}

The authors declare that they have no conflicts of interest.

\section{Acknowledgments}

This work was supported in part by National Natural Science Foundation of China (Grant No. 61901440), Beijing Municipal Natural Science Foundation (Grant No. 4202080), National Defense Basic Scientific Research Program (Grant No. SK2020-013-2), and One Hundred Person Project of the Chinese Academy of Sciences. The authors are grateful to the PRNet authors for opening their software source on the GitHub. The authors are also especially acknowledge Megvii Technology Limited for the Face++ expression recognition platform.

\section{References}

[1] H. Jin, X. Wang, Y. Lian, and J. Hua, "Emotion information visualization through learning of 3D morphable face model," The Visual Computer, vol. 35, no. 4, pp. 535-548, 2019.

[2] H. Qayyum, M. Majid, S. M. Anwar, and B. Khan, "Facial expression recognition using stationary wavelet transform features," Mathematical Problems in Engineering, vol. 2017, Article ID 9854050, 9 pages, 2017.

[3] K. Yan, W. Zheng, T. Zhang, Y. Zong, and Z. Cui, "Crossdatabase non-frontal facial expression recognition based on transductive deep transfer learning," IEEE Access, vol. 7, pp. 1-8, 2019.

[4] J. Shao and Y. Qian, "Three convolutional neural network models for facial expression recognition in the wild," Neurocomputing, vol. 355, pp. 82-92, 2019.

[5] P. Werner, F. Saxen, A. Al-Hamadi, and H. Yu, "Poster for "generalizing to unseen head poses in facial expression recognition and action unit intensity estimation"," in Proceedings of the 14th IEEE International Conference on Automatic Face \& Gesture Recognition (2019), Lille, France, 2019.

[6] A. Samanta and T. Guha, "On the role of head motion in affective expression," in Proceedings of the IEEE International
Conference on Acoustics, Speech and Signal Processing (ICASSP), pp. 2886-2890, New Orleans, LA, USA, March 2017.

[7] F. Malawski, B. Kwolek, and S. Sako, "Using kinect for facial expression recognition under varying poses and illumination," Active Media Technology, vol. 8610, pp. 395-406, 2014.

[8] H. Kaya, G. Furkan, S. Afshar, and A. A. Salah, "Contrasting and combining least squares based learners for emotion recognition in the wild," in Proceedings of the 2015 ACM on International Conference on Multimodal Interaction, pp. 459-466, Seattle, WA, USA, November 2015.

[9] S. P. Khandait, R. C. Thool, and P. D. Khandait, "Automatic facial feature extraction and expression recognition based on neural network," International Journal of Advanced Computer Science and Applications, vol. 2, no. 1, pp. 113-118, 2012.

[10] M. Iqtait, F. S. Mohamad, and M. Mamat, "Feature extraction for face recognition via active shape model (ASM) and active appearance model (AAM)," IOP Conference Series: Materials Science and Engineering, vol. 332, pp. 1-9, 2018.

[11] C. J. Lin, C. H. Lin, and S. H. Wang, "Multiple convolutional neural networks fusion using improved fuzzy integral for facial emotion recognition," Applied Sciences-Basel, vol. 9, no. 13, pp. 1-14, 2019.

[12] Y. Biao, C. Jinmeng, N. Rongrong, and Z. Yuyu, "Facial expression recognition using weighted mixture deep neural network based on double-channel facial images," IEEE Access, vol. 6, pp. 4630-4640, 2017.

[13] B.-F. Wu and C.-H. Lin, "Adaptive feature mapping for customizing deep learning based facial expression recognition model," IEEE Access, vol. 6, pp. 12451-12461, 2018.

[14] Y. Liu, J. Zeng, S. Shan, and Z. Zheng, "multi-channel poseaware convolution neural networks for multi-view facial expression recognition," in Proceedings of the 13th IEEE International Conference on Automatic Face \& Gesture Recognition (FG 2018), pp. 458-465, Xi'an, China, May 2018.

[15] X. Wang, X. Wang, and Y. Ni, "Unsupervised domain adaptation for facial expression recognition using generative adversarial networks," Computational Intelligence and Neuroscience, vol. 2018, Article ID 7208794, 10 pages, 2018.

[16] Y. H. Lai and S. H. Lai, "Emotion-preserving representation learning via generative adversarial network for multi-view facial expression recognition," in Proceedings of the 13th IEEE International Conference on Automatic Face \& Gesture Recognition (FG 2018), pp. 263-270, Xi’an, China, May 2018.

[17] V. Blanz and T. Vetter, "A morphable model for the synthesis of 3D faces," in Proceedings of the 26th Annual Conference on Computer Graphics and Interactive Techniques SIGGRAPH'99, pp. 187-194, July 1999.

[18] X. Zhu, Z. Lei, X. Liu, H. Shi, and S. Z. Li, "Face alignment across large poses: a 3D solution," in Proceedings of the 2016 IEEE Conference on Computer Vision and Pattern Recognition (CVPR), pp. 146-155, Las Vegas, NV, USA, 2016.

[19] Y. Feng, F. Wu, X. Shao, Y. Wang, and X. Zhou, "Joint 3D face reconstruction and dense alignment with position map regression network," in Proceedings of the 15th European Conference on Computer Vision, vol. 11218, Munich, Germany, 2018.

[20] P. M. Bentler and C.-P. Chou, "Practical issues in structural modeling," Sociological Methods \& Research, vol. 16, no. 1, pp. 78-117, 1987.

[21] K. Wang, X. Peng, J. Yang, D. Meng, and Y. Qiao, "Region attention networks for pose and occlusion robust facial expression recognition," IEEE Transactions on Image Processing, vol. 29, pp. 4057-4069, 2020. 
[22] Y. Li, J. Zeng, S. Shan, and X. Chen, "Occlusion aware facial expression recognition using CNN with attention mechanism," IEEE Transactions on Image Processing, vol. 28, no. 5, pp. 2439-2450, 2019.

[23] M. N. Riaz, Y. Shen, M. Sohail, and M. Guo, "eXnet: an efficient approach for emotionrecognition in the wild," Sensors (Basel, Switzerland), vol. 20, no. 4, pp. 1-17, 2020.

[24] J. Cai, Z. Meng, S. K. Ahmed, Z. Li, O. R. James, and Y. Tong, "Island loss for learning discriminative features in facial expression recognition," in Proceedings of the 2018 13th IEEE International Conference on Automatic Face \& Gesture Recognition (FG 2018), pp. 302-309, Xi'an, China, May 2018.

[25] Z. Meng, P. Liu, J. Cai, S. Han, and Y. Tong, "Identity-aware convolutional neural network for facial expression recognition," in Proceedings of the 2017 12th IEEE International Conference on Automatic Face \& Gesture Recognition (FG 2017), pp. 558-565, IEEE, Washington, DC, USA, May-June 2017.

[26] D. L. Jackson, "Revisiting sample size and number of parameter estimates: some support for the $N: q$ hypothesis," Structural Equation Modeling: A Multidisciplinary Journal, vol. 10, pp. 128-141, 2013.

[27] R. Willinger, H.-S. Kang, and B. Diaw, “Three-dimensional human head finite-element model validation against two experimental impacts," Annals of Biomedical Engineering, vol. 27 , no. 3, pp. 403-410, 1999.

[28] F. C. Jeffrey, A. Zara, and E. Paul, "Running head: facial action coding system observer-based measurement of facial expression with the facial action coding system," in Proceedings of the 43rd Annual 2009 International Carnahan Conference on Security Technology, Zurich, Switzerland, 2009.

[29] K. Waters, "A muscle model for animating three-dimensional facial expression," in Proceedings of the 14st Annual Conference on Computer Graphics and Interactive Techniques, vol. 21, pp. 17-24, Anaheim, CA, USA, 1987.

[30] S. Schaefer, T. Mcphail, and J. Warren, "Image deformation using moving least squares," ACM Transactions on Graphics, vol. 25 , no. 3, pp. 533-540, 2006.

[31] E. S. Yus and P. Simon, "Striated muscle," The American Journal of Dermatopathology, vol. 22, no. 6, pp. 503-509, 2000.

[32] D. S. Smith, "Reticular organizations within the striated muscle cell," The Journal of Biophysical and Biochemical Cytology, vol. 10, no. 4, pp. 61-87, 1961.

[33] B. Aronov, S. Har-Peled, C. Knauer, Y. Wang, and C. Wenk, "Fréchet distance for curves, revisited," Computer Science, vol. 14, pp. 52-63, 2006. 